\title{
On the probability distribution function of small-scale interplanetary magnetic field fluctuations
}

\author{
R. Bruno ${ }^{1}$, V. Carbone ${ }^{2}$, L. Primavera $^{2}$, F. Malara ${ }^{2}$, L. Sorriso-Valvo ${ }^{2}$, B. Bavassano ${ }^{1}$, and P. Veltri ${ }^{2}$ \\ ${ }^{1}$ Istituto Fisica Spazio Interplanetario del CNR, 00133 Roma, Italy \\ ${ }^{2}$ Dipartimento di Fisica Università della Calabria, 87036 Rende (Cs), Italy
}

Received: 10 May 2004 - Revised: 8 July 2004 - Accepted: 16 August 2004 - Published: 3 November 2004

\begin{abstract}
In spite of a large number of papers dedicated to the study of MHD turbulence in the solar wind there are still some simple questions which have never been sufficiently addressed, such as:

a) Do we really know how the magnetic field vector orientation fluctuates in space? b) What are the statistics followed by the orientation of the vector itself? c) Do the statistics change as the wind expands into the interplanetary space?

A better understanding of these points can help us to better characterize the nature of interplanetary fluctuations and can provide useful hints to investigators who try to numerically simulate MHD turbulence.

This work follows a recent paper presented by some of the authors which shows that these fluctuations might resemble a sort of random walk governed by Truncated Lévy Flight statistics. However, the limited statistics used in that paper did not allow for final conclusions but only speculative hypotheses. In this work we aim to address the same problem using more robust statistics which, on the one hand, forces us not to consider velocity fluctuations but, on the other hand, allows us to establish the nature of the governing statistics of magnetic fluctuations with more confidence.

In addition, we show how features similar to those found in the present statistical analysis for the fast speed streams of solar wind are qualitatively recovered in numerical simulations of the parametric instability. This might offer an alternative viewpoint for interpreting the questions raised above.
\end{abstract}

Key words. Interplanetary physics (interplanetary magnetic fields) - Space plasma physics (waves and instabilities)

\section{Introduction}

The first turbulent model proposed by Kolmogorov (1941) didn't take into account that the rate of energy transfer along the turbulent cascade might not be scale-independent (Landau's objection). This situation, in the framework of a classi-

Correspondence to: R. Bruno

(bruno@ifsi.rm.cnr.it) cal Richardson's cascade, can be described by the fact that smaller and smaller eddies are less and less space-filling. In other words, turbulence would be unevenly or intermittently distributed in space. As a matter of fact, we address this phenomenon as Intermittency. Evidence of the presence of this phenomenon, when performing a statistical study on a generic fluctuating field $V(x)$, is that the probability distribution functions (PDF hereafter) of the differences $\delta v_{l}(x)=|V(x+l)-V(x)|$ normalized to the $\sigma$ of the distribution at scale $l$ do not rescale for different scales (Van Atta and Park, 1975).

In particular, the tails of these PDFs become more and more stretched at smaller and smaller scales. This means that the wings of the distributions become fatter and fatter. Such behaviour implies that, at smaller scales, extreme events become statistically more probable than if they were normally distributed.

Intermittency has also been found in the solar wind fluctuations, as demonstrated in the first studies performed by Burlaga (1991) in the outer heliosphere. In particular, this author showed an unexpected similarity between interplanetary observations on scales of $1 \mathrm{AU}$ and observations on scales of meters obtained for laboratory turbulence by Anselmet et al. (1984). These results suggested the universality of this phenomenon, which was independent on scale. On the other hand, Marsch and Liu (1993) and Carbone et al. (1995) were the first authors to study Intermittency in the inner heliosphere. In particular, the former authors showed the different intermittent character of fast and slow wind while the latter ones showed a possible first evidence for the Kraichnan scaling (Kraichnan, 1965) in a magnetofluid like the solar wind. Since then, several papers (Ruzmaikin et al., 1995; Tu et al., 1996; Horbury et al., 1997; Sorriso-Valvo et al., 1999; Bruno et al., 1999; Pagel and Balogh , 2003; Bruno et al., 2003) among others followed these first approaches to the problem of understanding Intermittency in the solar wind. However, novel techniques based on the properties of wavelets introduced by Farge et al. (1990) and first used within ordinary fluid dynamics by Onorato et al. (2000) and within the solar wind context by Veltri and Mangeney (1999), Bruno et al. (1999) and Bruno et al. (2001), represented a powerful 
tool to finally disclose the nature of intermittent events. In this last paper, the authors showed that the intermittent event they were able to single out was a so-called current-sheetassociated TD, as defined by Ho et al. (1995). This type of structure is associated with wind velocity gradients, a rapid magnetic field intensity change and a reversal of the maximum variance magnetic field component across the discontinuity, which was interpreted as the interface between two adjacent flux tubes, i.e. two regions characterized by different plasma and magnetic field conditions although within the same large-scale plasma region. During these studies, it was also noticed that the jumps performed by the tips of magnetic and velocity vectors resemble a typical Lévy process (Bruno et al., 2004). This process is similar to a random walk but the statistics governing the spatial jumps are characterized by extreme behaviour. Consequently, the presence of long-range correlations makes the Gaussian statistics, which govern the Brownian motion, no longer representative of the physical process. In fact, the spatial distribution of the directions assumed by these vectors during the selected time interval was not uniform but rather patchy. This particular behavior indicates the presence of particular directions along which the fluctuating field vector would roughly remain aligned for a longer time. Then, a rapid and large jump would characterize the transfer from one patch to another. These large jumps were recognized to make the fluctuating field more intermittent. Moreover, the highly Alfvénic character of the selected time interval clearly showed that propagating modes and coherent structures were both contributing to the observed turbulence and strengthened the already proposed view of a turbulence made of a mixture of waves and structures (Matthaeus et al., 1990; Bruno and Bavassano, 1991; Tu and Marsch, 1991; Marsch and Liu, 1993; Tu and Marsch, 1993; Klein et al., 1993, among others). In reality, it took a long time to reach this view of interplanetary MHD turbulence. As a matter of fact, the first spectra of solar wind fluctuations obtained from the observations of Mariner 2 in 1962. were interpreted by Coleman (1968) as evidence for the presence of turbulent processes, possibly MHD turbulence as described by Kraichnan (1965). The velocity shear mechanism proposed by Coleman (1968) would be sustained by strong velocity gradients present in the solar wind and would produce large-scale Alfvén waves that would transfer their energy to smaller and smaller scales through a turbulent process. On the other hand, Belcher and Davis (1971), looking at the correlation between velocity and magnetic field fluctuations observed by Mariner 5, concluded that interplanetary fluctuations were exclusively made of outward propagating Alfvén modes, mostly of solar origin. Obviously, these two points of view were in contradiction because the absence of inward propagating modes would preclude the development of a turbulent cascade of energy to form a spectrum similar to the one observed by Coleman (1968). In reality, interplanetary fluctuations are not just Alfvén waves. Only several years later, a careful data analysis performed on the observations provided by the Helios spacecraft contributed to make the idea of a possible coexistence of propagating waves and convected structures accepted (see review by Tu and Marsch, 1995). Moreover, only recently, theoretical efforts (Wu and Chang ,2000; Vasquez and Hollweg, 2004; Vasquez et al., 2004) have shown the possibility that propagating modes and coherent structures might share a common origin within the general view described by the physics of complexity. Propagating modes would experience resonances which generate coherent structures which, in turn, will migrate, interact and eventually generate new modes. Moreover, Primavera et al. (2003), using a 1-D MHD numerical simulations based on a pseudo-spectral code, were able to qualitatively reproduce the radial behavior of magnetic field and velocity Intermittency observed by Bruno et al. (2003) in the inner heliosphere. In particular, they numerically simulated the propagation of a turbulent Alfvénic spectrum in a uniform background magnetic field. As a matter of fact, coherent structures were created during the spectral evolution due to the parametric instability resembling a sort of shocklet or current sheet. Obviously, the model has strong limitations since while the 1-D code allows for dependence on only 1 spatial coordinate, the vectors can have all 3 Cartesian components. As a consequence, these results remain at a qualitative level.

Using the idea of McCracken and Ness (1966) as a starting point, Bruno et al. (2001) proposed the spaghetti-like model in which Alfvénic fluctuations propagate within a convected structure made of tangled flux tubes, each of them being characterized by their local magnetic field and plasma. These structures represent the correlated part of the signal, unlike from the Alfvénic component which does not conserve spatio-temporal coherence.

Moreover, it was found that the statistics associated with these fluctuations experienced a strong radial evolution. As a matter of fact, directional jumps, within fast solar wind, evolved from a more Gaussian-like statistic at $0.3 \mathrm{AU}$ towards a sort of Truncated Lévy Flight (Mantegna, 1994) statistic at $0.9 \mathrm{AU}$. This phenomenon suggested that the possible cause of the radial evolution was the radial progressive depletion of the Alfvénic component of the turbulent fluctuations with respect to the convected structure. However, the limited statistics due to the low resolution of plasma data did not allow for a more refined analysis. In this paper we used higher resolution data, about one order of magnitude higher in frequency, to study in more detail the structure of the PDFs of directional fluctuations but we had to limit this study to the only magnetic component of the fluctuations.

\section{Data analysis}

Data used in this work are 6-s averages of magnetic field measurements performed by Helios $2 \mathrm{~s} / \mathrm{c}$ during its primary mission to the Sun almost three decades ago, in 1976. This is the only data set available covering the heliocentric distance range between 0.3, the closest approach to the Sun, and $1 \mathrm{AU}$. So far, this data set has been extremely valuable to study the main physical mechanisms governing the solar wind turbulence. In particular, it has been very useful 


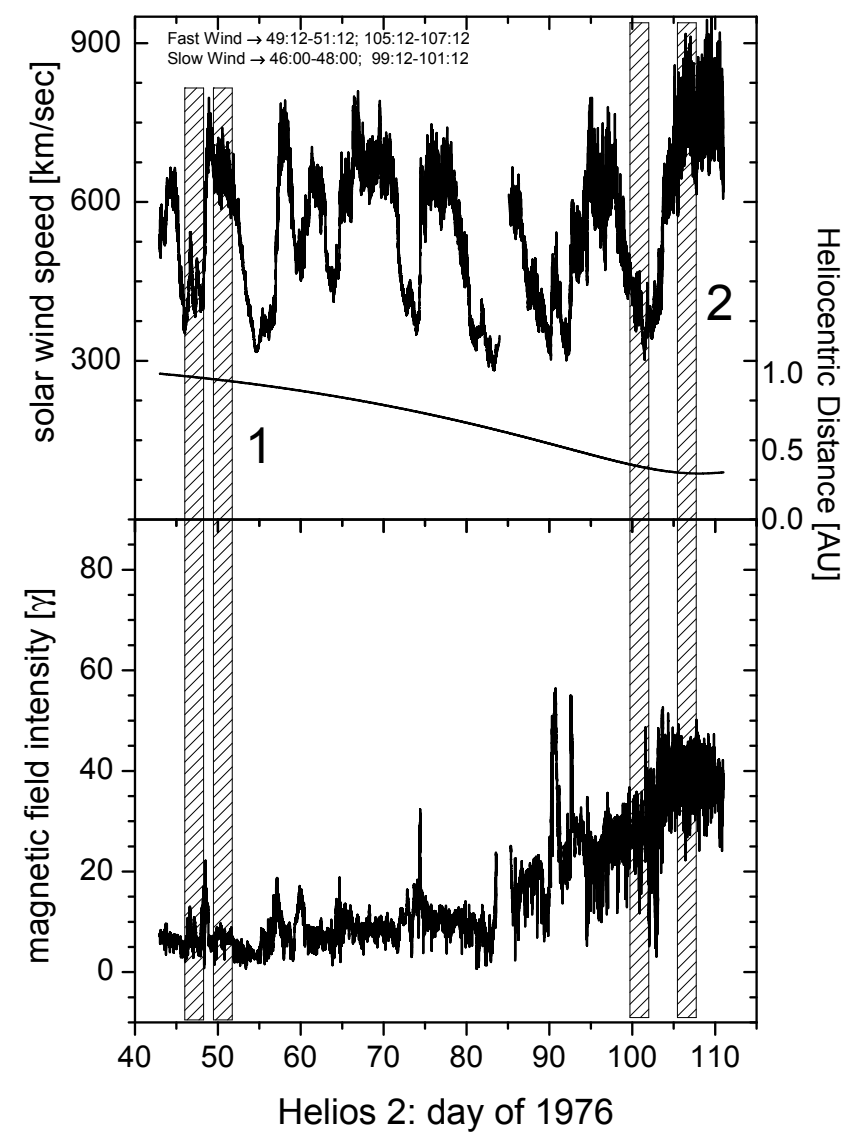

Fig. 1. Top panel: solar wind speed profile versus time as recorded during the first solar mission of Helios 2. The solid, smooth line represents the heliocentric distance of the $\mathrm{s} / \mathrm{c}$ which varied between 0.97 and 0.29 AU. Bottom panel: magnetic field intensity profile versus time. The whole interval was characterized by the presence of only one shock around day 90 . Vertical hatched regions identify time intervals chosen for this analysis. There are two high speed intervals located in the trailing edge of two corotating streams and two low speed intervals ahead of them.

to study the radial evolution of interplanetary turbulence. As a matter of fact, this data set contains in-situ observations at different heliocentric distances of magnetic field and plasma belonging to the same corotating solar source region of high velocity wind. Thus, the stationary character of this region allowed for studies which could focus on the radial evolution of solar wind turbulence, providing extremely important results which have been reviewed in an excellent paper by $\mathrm{Tu}$ and Marsch (1995). In the present analysis we will refer to this particular high velocity stream and to the low velocity wind ahead of it, observed at two different heliocentric distances, namely 0.3 and 0.9 AU. These time intervals are highlighted in Fig. 1 by vertical hatched stripes which, from the top to the bottom of the figure, go across velocity and heliocentric distance in the top panel and magnetic field intensity in the bottom panel. Each of the four time intervals lasts 2 days and has approximately 28800 6-s averages, taking into account data gaps. Temporal extremes, average heliocentric
Table 1. Time extremes and average values characterizing the intervals.

\begin{tabular}{cccc}
\hline $\begin{array}{c}\text { time interval } \\
\text { (dd:hh) }\end{array}$ & $\begin{array}{c}\text { distance } \\
(\mathrm{AU})\end{array}$ & $\begin{array}{c}<V> \\
(\mathrm{km} / \mathrm{s})\end{array}$ & $\begin{array}{c}<B> \\
(\mathrm{nT})\end{array}$ \\
\hline 46:00-48:00 & 0.90 & 433 & 6.8 \\
49:12-51:12 & 0.88 & 643 & 6.8 \\
99:12-101:12 & 0.34 & 405 & 28.9 \\
105:12-107:12 & 0.29 & 729 & 42.1 \\
\hline
\end{tabular}

distance, average wind speed and field intensity are reported in Table 1 for each interval.

Just for sake of completeness we like to add that the same corotating stream was also observed at 0.7 AU starting around day 74 but we will omit that time interval since it would be redundant for the analysis we present here. As already stated in Sect. 1, the main goal of this work is the study and characterization of directional fluctuations. To do so, we start with plotting the position of the tip of the magnetic field vector within the reference system of the three coordinate axes during sub-intervals of only 2000 points within the selected time periods. These sub-intervals can be considered representative of each particular interval they refer to. A longer sequence of points would make it impossible, in the graphical format we used, to recognize the differences between different intervals.

In Fig. 2 we show data relative to fast wind observed at 0.3 and $0.9 \mathrm{AU}$ in the top and bottom panels, respectively. In this graphical representation, the origin of the coordinated axes is right in the center of the 3-D plot. This means that, in the case of a vector of constant magnitude which changes direction in a random way, after sufficient time, we would see a dark sphere centered in the middle of the graph with a radius equal to the vector magnitude. In case directional fluctuations were concentrated around some particular direction we would expect the surface of the sphere to be unevenly covered. In the top panel, the tip of the vector wanders in a random way on the surface of almost half a sphere without showing any preferential direction. The enhanced regularity of this surface, if compared to slow wind in the following, depends on the fact that, during this high velocity stream, the field intensity is quite constant, as generally expected for a high velocity stream. One of the main features of this stream is that it is highly Alfvénic, as already stressed several times in the available literature (see, for example, the paper by Bruno et al., 1985). This fact, obviously, induces large directional fluctuations on the direction of the ambient magnetic field. As a consequence, the dark spot in the figure completely covers half the sphere. Obviously, only half of the sphere is covered since magnetic field polarity remains generally constant within high velocity streams while it can flip from positive to negative and vice versa more easily within a slow wind. However, when we reach $0.9 \mathrm{AU}$ the situation evolves dramatically. Although the tip of the vector 


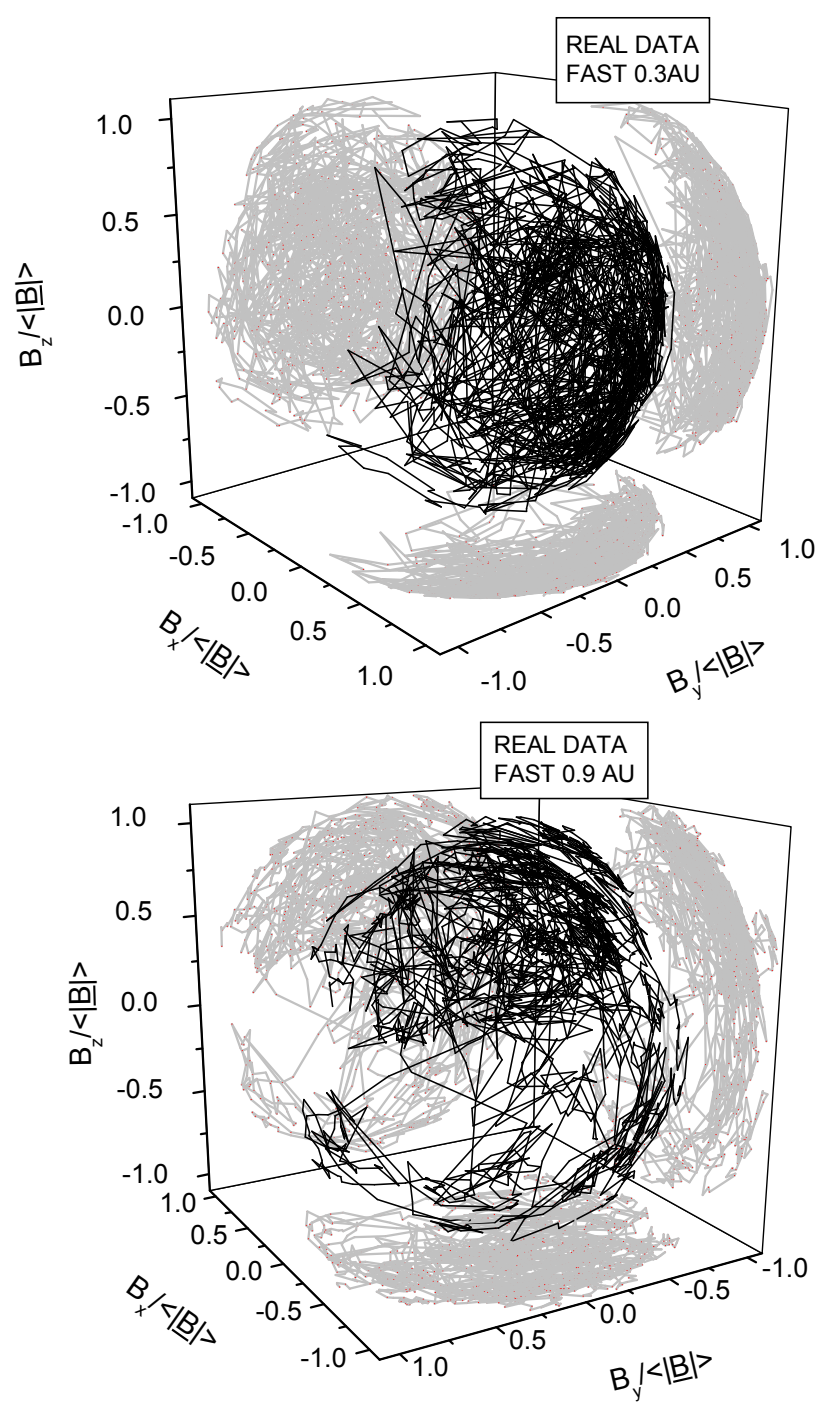

Fig. 2. The top panel refers to a fast wind recorded at $0.3 \mathrm{AU}$ while the bottom panel refers to a fast wind at $0.9 \mathrm{AU}$. Each point of both plots represents the location of the tip of the magnetic field vector for each 6-s average. These locations have then been connected by a black straight line to form a trajectory. Moreover, the shadow of this trajectory is also shown on the three coordinate planes to better understand the spatial 3-D configuration. In these panels we show only intervals of 2000-point representing larger intervals of 28800 6-s averages. Values of each component have been normalized to the average magnetic field intensity measured within the 2000 points interval. The top panel shows a more uniform coverage with respect to the bottom panel, which shows a sort of patchy configuration. In other words, there is some kind of evolution during the radial expansion which is dramatically reflected in this kind of spatial behaviour.

still wanders on the surface of only half a sphere, it does not cover this surface completely but leaves out wide areas. The distribution of the dark spots suggests that the presence of preferred spatial directions, connected by large and quick jumps which take only a few data points, begins to emerge as the heliocentric distance increases.
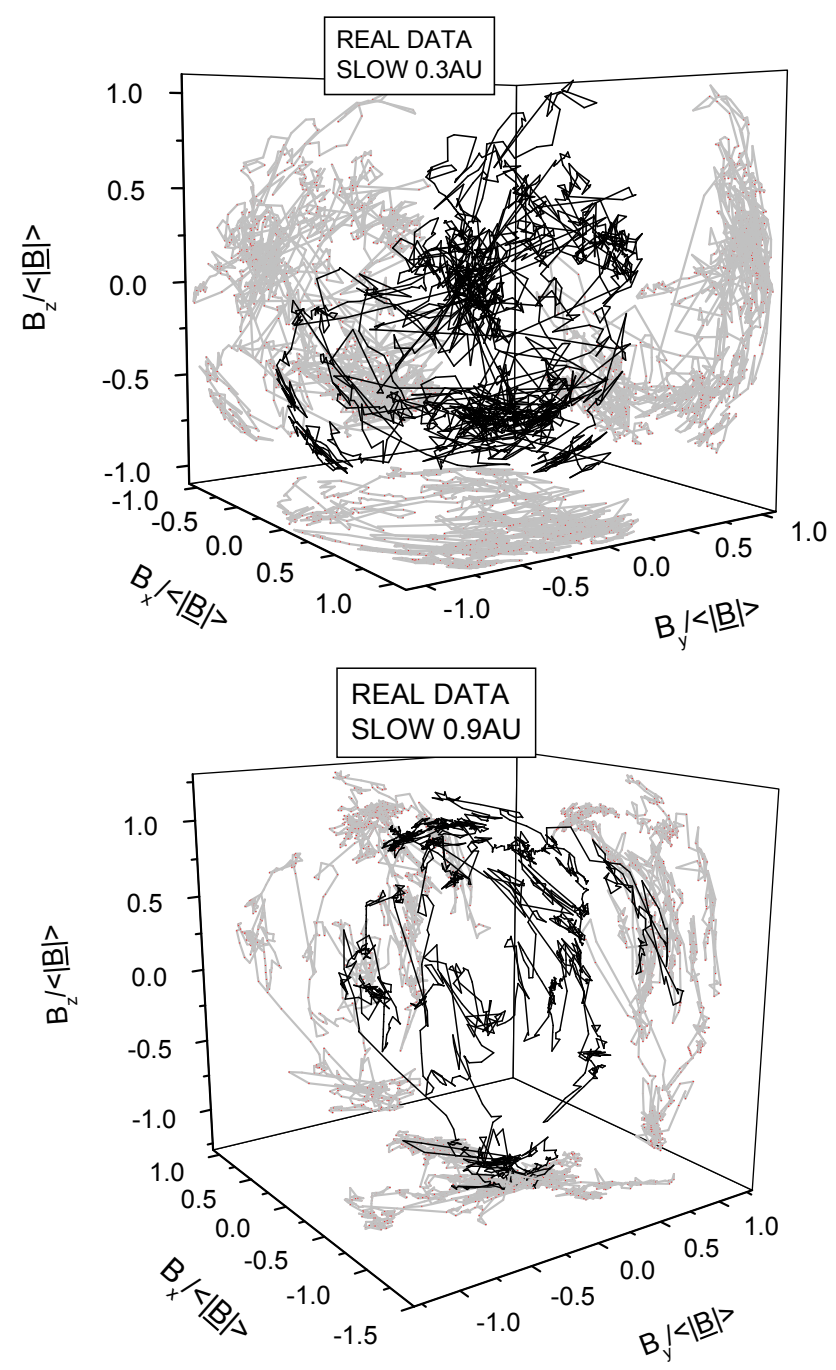

Fig. 3. The top panel refers to a slow wind recorded at $0.3 \mathrm{AU}$ while the bottom panel refers to slow wind at $0.9 \mathrm{AU}$. The format is the same used for Fig. 2 and, also in this case, we show only 2000 points out of 28800 points of each selected time interval. Both configurations relative to 0.3 and $0.9 \mathrm{AU}$ greatly differ from what we observed within fast wind. In both cases a patchy configuration is clearly visible, meaning that the tip of the vector dawdles longer around some particular orientation. At first sight, the two panels show similar configurations, although fluctuations at $0.3 \mathrm{AU}$ appear to be slightly larger. Definitely, we do not observe the same radial evolution noticed for a fast wind.

On the contrary, the evolution we just noticed in a fast wind is much less dramatic within a slow wind, as shown in the two panels of Fig. 3. The top panel, which refers to observations recorded at $0.3 \mathrm{AU}$, clearly shows the presence of two main directions around which the magnetic field vector fluctuations mainly cluster. Moreover, as in the previous case, large jumps connecting one spot to the other are clearly visible. When we move to $0.9 \mathrm{AU}$ in the lower panel, we do not notice much of a difference with respect to the situation encountered in the upper panel. Fluctuations appear to be generally smaller but the spotty configuration clearly emerges again. 
However, this kind of graphical representation is not sufficient to give an idea of how the tip of the vector really moves in time unless complemented by the information we provide in the top panel of Fig. 4. In this panel we show the vector displacement $|\delta \underline{B}(t)|$, normalized to $\langle|\underline{B}|>$, between each $\underline{B}(t)$ and an arbitrary fixed direction which we chose to be the direction of the first vector $\underline{B}\left(t_{0}\right)$ of the time series. Thus, following this definition, each individual $|\delta \underline{B}(t)|$ is given by:

$$
|\delta \underline{B}(t)|=\sqrt{\sum_{i=x, y, z}\left(B_{i}(t)-B_{i}\left(t_{0}\right)\right)^{2}} .
$$

This time sequence, the same used for the top panel of Fig. 3 which refers to a slow wind at $0.3 \mathrm{AU}$, clearly shows a small amplitude and high frequency fluctuations superimposed on a sort of larger amplitude low-frequency background structure. This background structure is characterized by a few large and quick directional jumps. The effect of these jumps is to move the fluctuating vector from one particular average direction to another, i.e. from one dark spot to another (Fig. 3). This type of information, together with the 3-D graphical representation, gives an idea of how the vector direction really fluctuates in space and time. Moreover, most of the time the largest directional jumps are associated with the largest changes in the field intensity (bottom panel). So, these two panels suggest that during short time intervals the field can be characterized by a most probable orientation and a most probable intensity. In other words, these regions appear to be distinguishable from each other and the transition from one to another is through a large rotational jump and a change in the field intensity. Similar findings have already been reported in a previous paper (Bruno et al., 2001), although it was focused on a single case study and on larger scales. In that same study it was found that this kind of transition, or border, was a tangential discontinuity not in pressure balance. The features we notice in the present study might be TDs as well, although we cannot prove it since we don't have plasma data with the same time resolution of magnetic field data. If this is the case, the structure we have seen at larger scales replicates at smaller scales in a kind of self-similar manner.

Results relative to a slow wind at $0.9 \mathrm{AU}$ are shown in Fig. 5 in the same format as the previous figure. Although, both panels show fluctuations generally smaller than the corresponding ones observed at $0.3 \mathrm{AU}$, corresponding features in both profiles are still clearly recognizable. Thus, radial evolution within slow wind doesn't play much of an influence on this kind of relationship.

In Fig. 6 we show vector displacement for the time interval recorded at $0.3 \mathrm{AU}$, within a fast wind in the same format as the previous two figures. Directional fluctuations appear to be very chaotic and not as much structured as we found in the slow wind. Thus, it is certainly more difficult to recognize structures similar to the ones observed in the previous figures and correlate them to the profile of the magnetic field intensity in the bottom panel. As a matter of fact, we expect to find large amplitude directional fluctuations within a fast
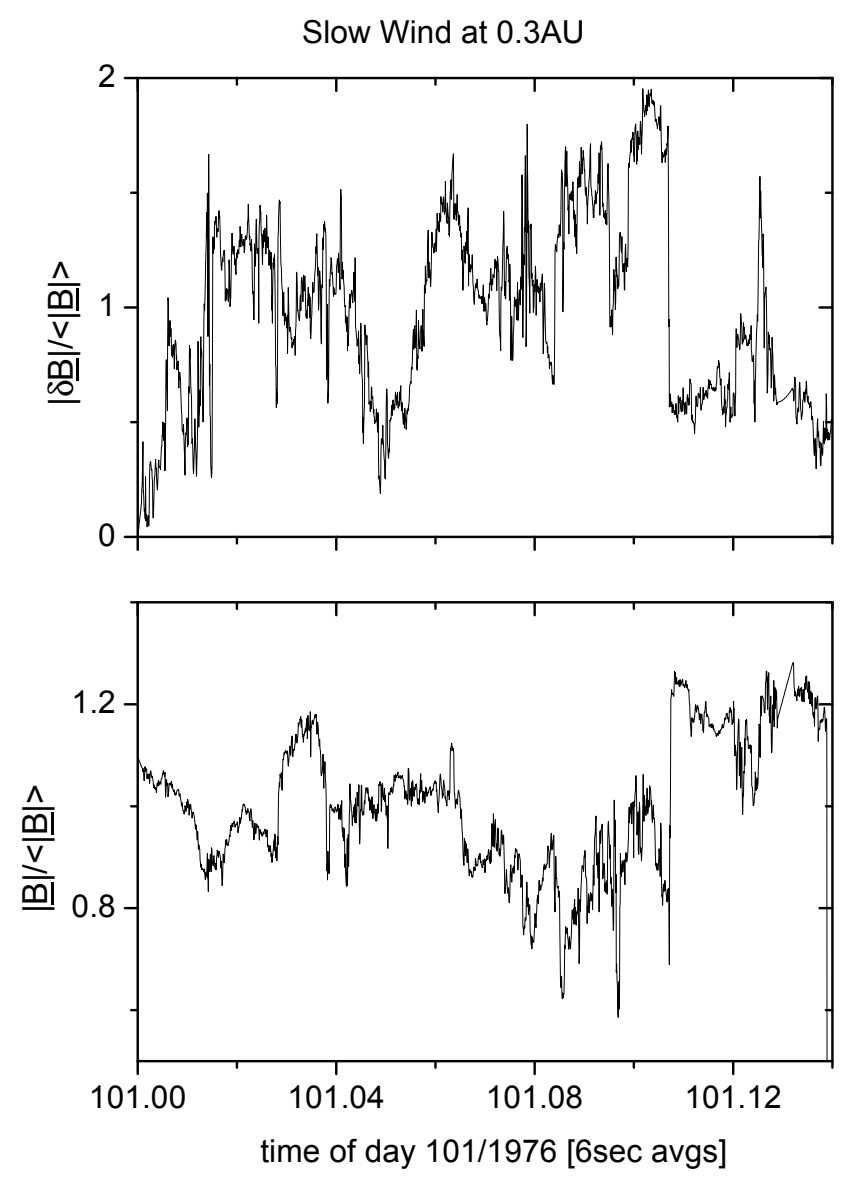

Fig. 4. Data refer to a slow wind at $0.3 \mathrm{AU}$. Top panel: vector displacement $|\delta \underline{B}(t)|$, normalized to $\langle|\underline{B}|>$, between each $\underline{B}(t)$ and an arbitrary fixed direction versus time. The arbitrary direction was chosen as the direction of the first vector of the time series. This kind of graph shows a series of time intervals during which the vector displacement tends to remain approximately close to the average level. These time intervals are interleaved by large and quick directional jumps. Moreover, the largest jumps often coincide with remarkable changes in field intensity as shown in the bottom panel.

wind, especially close to the Sun, because we are aware of the relevant presence of Alfvénic modes in this type of wind. As a consequence, we believe that these fluctuations mask the correspondence we were able to highlight within a slow wind which, a priori, might be similar to that. Consequently, if the Alfvénic modes had a smaller amplitude, we would be able to recognize and relate similar features in both panels.

As a matter of fact, results shown in Fig. 7 relative to fast wind data recorded at $0.9 \mathrm{AU}$ strongly support our intuition. The top panel of this figure shows a well structured profile, not much different from that observed in a slow wind. This time, several structures noticed in the top panel can easily be related to companion structures in the bottom panel. The reason lies in the fact that the amplitude of Alfvénic fluctuations is largely reduced at the Earth's orbit compared to short heliocentric distances (see the wide related literature in Tu and Marsch, 1995). 

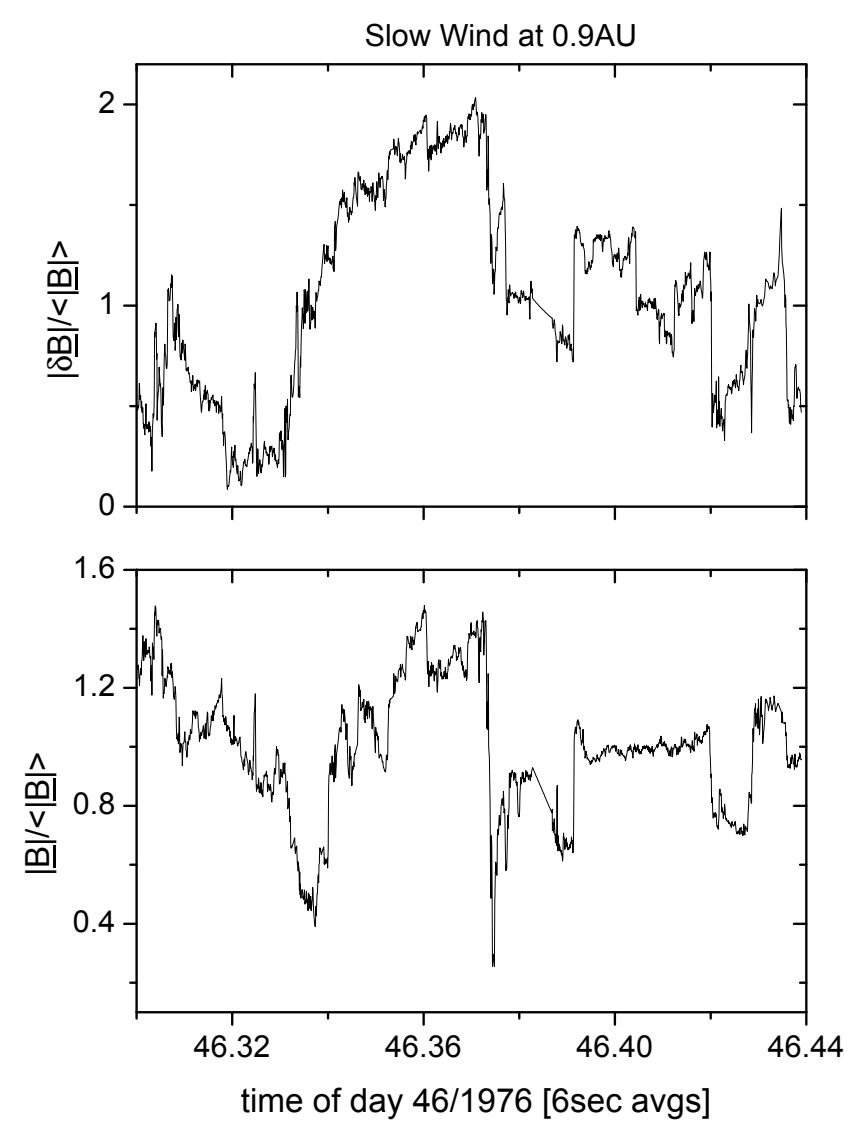

Fig. 5. Vector displacement versus time in the same format as of Fig. 4 relative to a slow wind at $0.9 \mathrm{AU}$. Largest vector displacements (top panel) often coincide with large compressive events, as shown in the bottom panel. As shown in the paper, the statistics of these vector displacements is remarkably similar to that shown in the previous Fig. 4

This qualitative study has to be substantiated with some quantitative evaluation of the relative importance of these two components contributing to the observed interplanetary turbulence. To do so, in the following, we will discuss and compare the probability distribution functions (PDF) of the directional fluctuations observed within each time interval. In order to look for a possible scaling between different PDFs, we have normalized each $|\delta \underline{B}(t)|$ to the standard deviation $\sigma$ of the relative distribution. Moreover, the maximum amplitude of each PDF was normalized to 1. In Fig. 8 we show PDFs for the fast wind samples recorded at 0.3 and $0.9 \mathrm{AU}$ in the top and bottom panels, respectively. We found that both distributions can be reasonably well fitted by a double lognormal distribution in the form reported by Eq. (2).

$$
\begin{aligned}
& P(\xi)=\frac{A_{1}}{\sigma_{1} \xi \sqrt{2 \pi}} \exp \left[-\left(\frac{\ln \left|\xi / \delta_{1}\right|}{\sqrt{2} \sigma_{1}}\right)^{2}\right]+\frac{A_{2}}{\sigma_{2} \xi \sqrt{2 \pi}} \\
& \quad \exp \left[-\left(\frac{\ln \left|\xi / \delta_{2}\right|}{\sqrt{2} \sigma_{2}}\right)^{2}\right]
\end{aligned}
$$
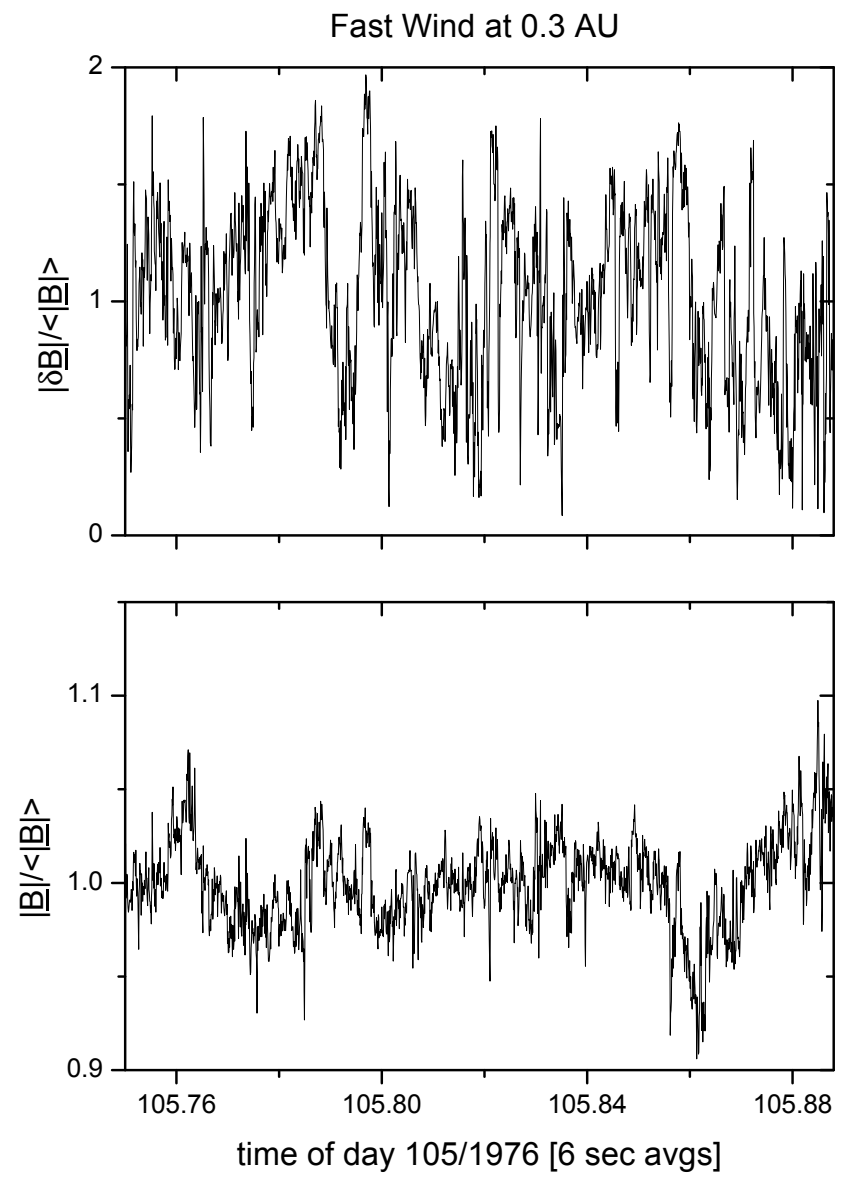

Fig. 6. Fast wind at $0.3 \mathrm{AU}$. Normalized vector displacements versus time in the same format as Figs. 4 and 5, shown in the top panel, while normalized vector intensities are shown in the bottom panel. The top panel shows large fluctuations which are difficult to relate to the profile of the magnetic field intensity in the bottom panel, although some corresponding events can still be recognized. In this sense, fast wind at $0.3 \mathrm{AU}$ differs from the slow wind samples we already discussed.

where $\xi, \delta, \sigma>0$. The variable $\xi$ stands for the different $\left|\delta \underline{B}_{i}\right| / \sigma$, one for each bin of the distribution, $A_{i}$ is a measure of the area under each curve, $\delta_{i}$ is called a scale parameter and represents the median, $\sigma_{i}$ is the shape parameter. Larger values of $\sigma_{i}$ push the x-location of the peak of the distribution towards lower values. Obviously, using a larger number of lognormals would provide a better fit but, the real conspicuous improvement is obtained only when we use two lognormals instead of just one.

The two lognormal components have a distinguishable role in the total PDF. One component accounts for the lognormal tail at large values of $|\delta \underline{B}| / \sigma$ while the second component takes care of the smallest values of $|\delta \underline{B}| / \sigma$. Moreover, taking into account the values of the parameters reported in Table 2 and relative to the best fit obtained reaching the minimum $\chi^{2}$ value for the two distributions at 0.3 and $0.9 \mathrm{AU}$, one of the two lognormals experiences a stronger radial evolution. In particular, the lognormal that represents fluctuations 
Fast Wind at 0.9AU
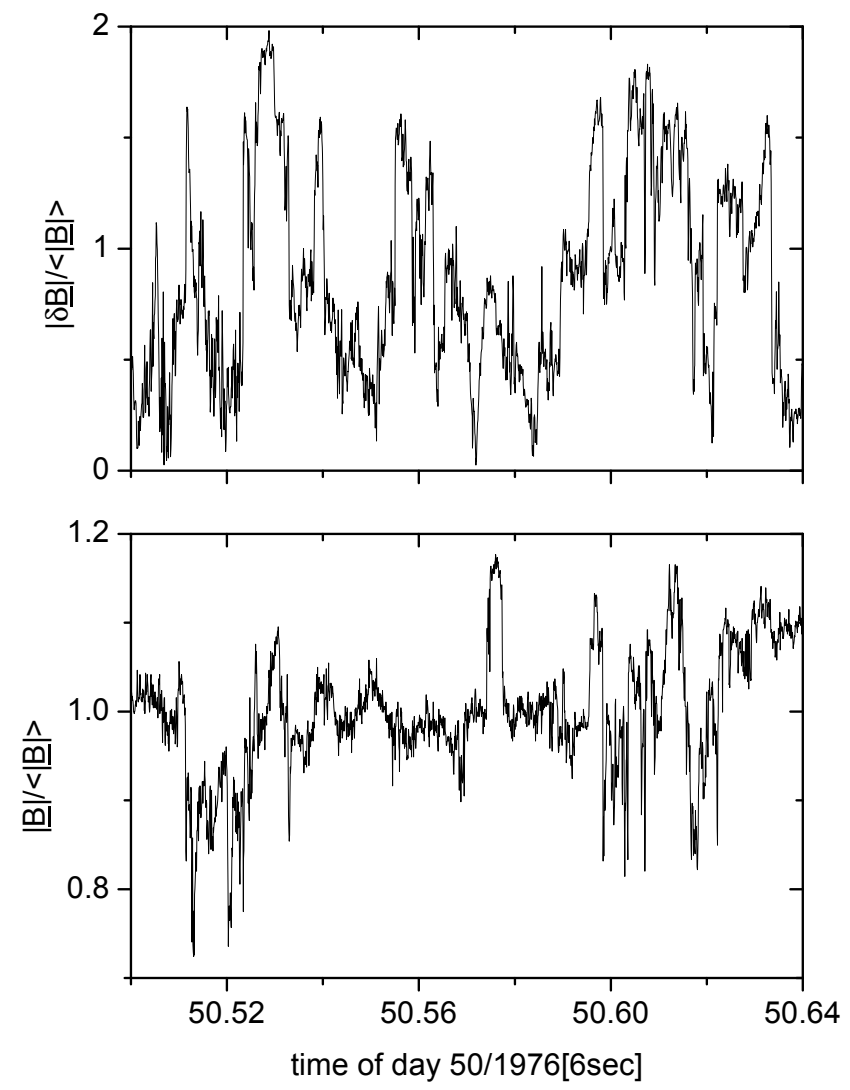

Fig. 7. Normalized vector displacements and normalized vector intensity versus time are shown in the same format of Figs. 4-6 for a fast wind at $0.9 \mathrm{AU}$. Vector displacements shown in the top panel appear to be less chaotic than those observed at $0.3 \mathrm{AU}$. A sort of underlying structure can be recognized and related to field intensity fluctuations shown in the bottom panel. In other words, this situation tends to resemble the one encountered within slow wind.

peaked on smaller $|\delta \underline{B}| / \sigma$ strongly decreases its contribution with increasing radial distance from the Sun. An estimate of this evolution can be inferred from the ratio of the areas $\mathrm{A}_{1}$ and $\mathrm{A}_{2}$ below each curve. While at $0.3 \mathrm{AU}$ the probability ratio $A_{2} / A_{1} \simeq 0.52$, at $0.9 \mathrm{AU}$ it drops to $\simeq 0.22$. Consequently, the contribution of the smaller PDF to the whole PDF varies from $34 \%$ at $0.3 \mathrm{AU}$ to $18 \%$ at $0.9 \mathrm{AU}$. All the other parameters do not experience a similar radial variation, and this behavior reflects in a depletion of the left-hand tail of the total PDF. However, it is worth noticing that both values of $\delta$ are located at somewhat larger values for the sample referring at $0.3 \mathrm{AU}$, suggesting that fluctuations are generally larger when closer to the Sun.

Similar conclusions apply to the PDFs relative to the angular fluctuations $\delta \alpha$ experienced by the vector orientation shown in Fig. 9. Obviously, this measure provides information only about directional fluctuations and is not influenced by compressive effects that may act on the vector intensity. As such, information contained in Fig. 9 is less meaningful than that discussed earlier but we like to show this kind of fig-

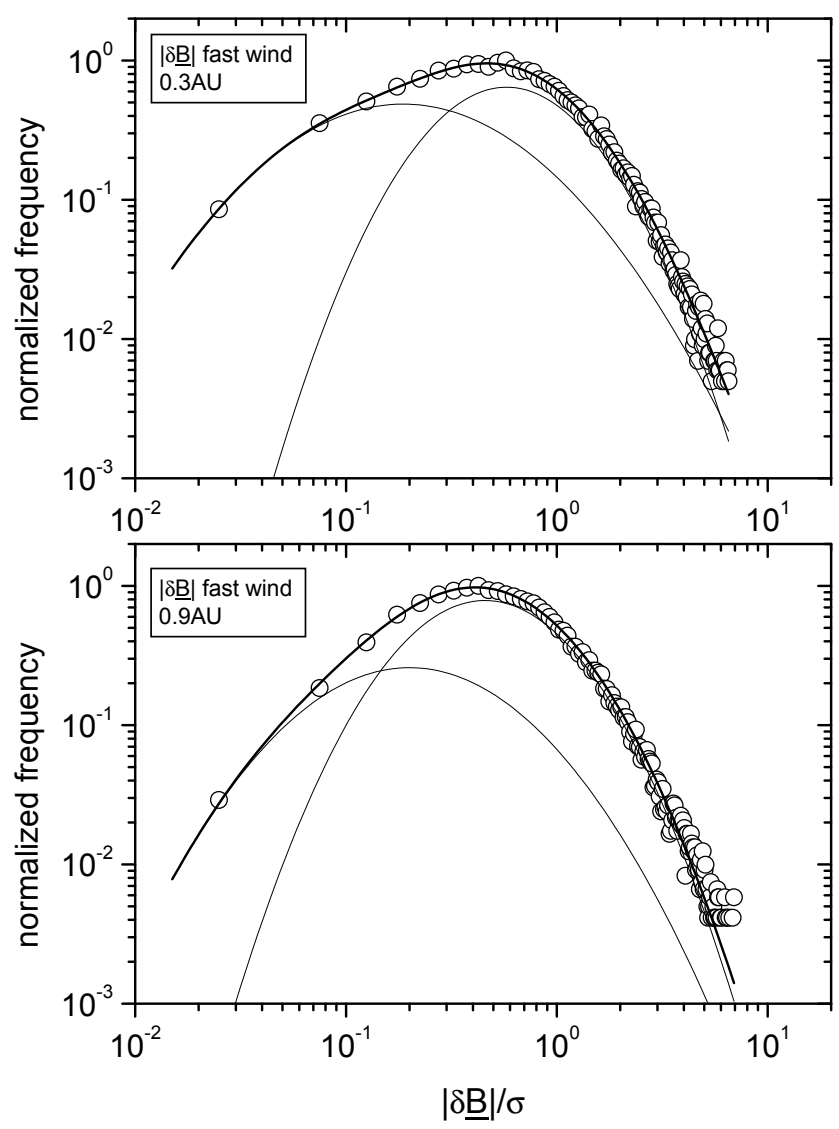

Fig. 8. PDFs of vector displacements $|\delta \underline{B}|$ normalized to $\sigma$ at 0.3 and $0.9 \mathrm{AU}$, for a fast wind, are shown in the top and bottom panels, respectively. The two thin solid curves refer to as many lognormals contributing to form the thick solid curve which best fit the distribution. Parameters relative to the fit are reported in Table 2.

ure, and the analogous one for a slow wind in Fig. 11, just for sake of completeness. Moreover, these distributions have not been normalized to their respective $\sigma$ valves since we like to show the effective angular range of these fluctuations. Also, for this fit we report the relative parameters which are shown in Table 3. For these fluctuations the ratio $\mathrm{A}_{2} / \mathrm{A}_{1}$ varies from $56 \%$ at $0.3 \mathrm{AU}$ to $40 \%$ at $0.9 \mathrm{AU}$. The PDF is clearly peaked at larger angles $\left(5.75^{\circ}\right.$ compared to $\left.2.25^{\circ}\right)$ at $0.3 \mathrm{AU}$ and its right tail reaches values close to $100^{\circ}$.

In Fig. 10 we show the vector displacement $|\delta \underline{B}|$ normalized to $\sigma$ for slow wind at 0.3 and $0.9 \mathrm{AU}$ in the same format as Fig. 8. The situation within a slow wind shows that radial evolution is almost absent. This can be inferred from the values of the best fit parameters reported in Table 4, which show that the contribution of the smaller lognormal can be neglected at both distances. As a matter of fact, the relative contribution of the smaller lognormal is between $0.26 \%$ at $0.3 \mathrm{AU}$ and $0.13 \%$ at $0.9 \mathrm{AU}$, respectively. Moreover, the values of the parameters relative to the larger lognormal only slightly change between 0.3 and $0.9 \mathrm{AU}$, suggesting that these fluctuations do not evolve much with radial distance, as expected for a slow wind. In summary, the constancy of all 

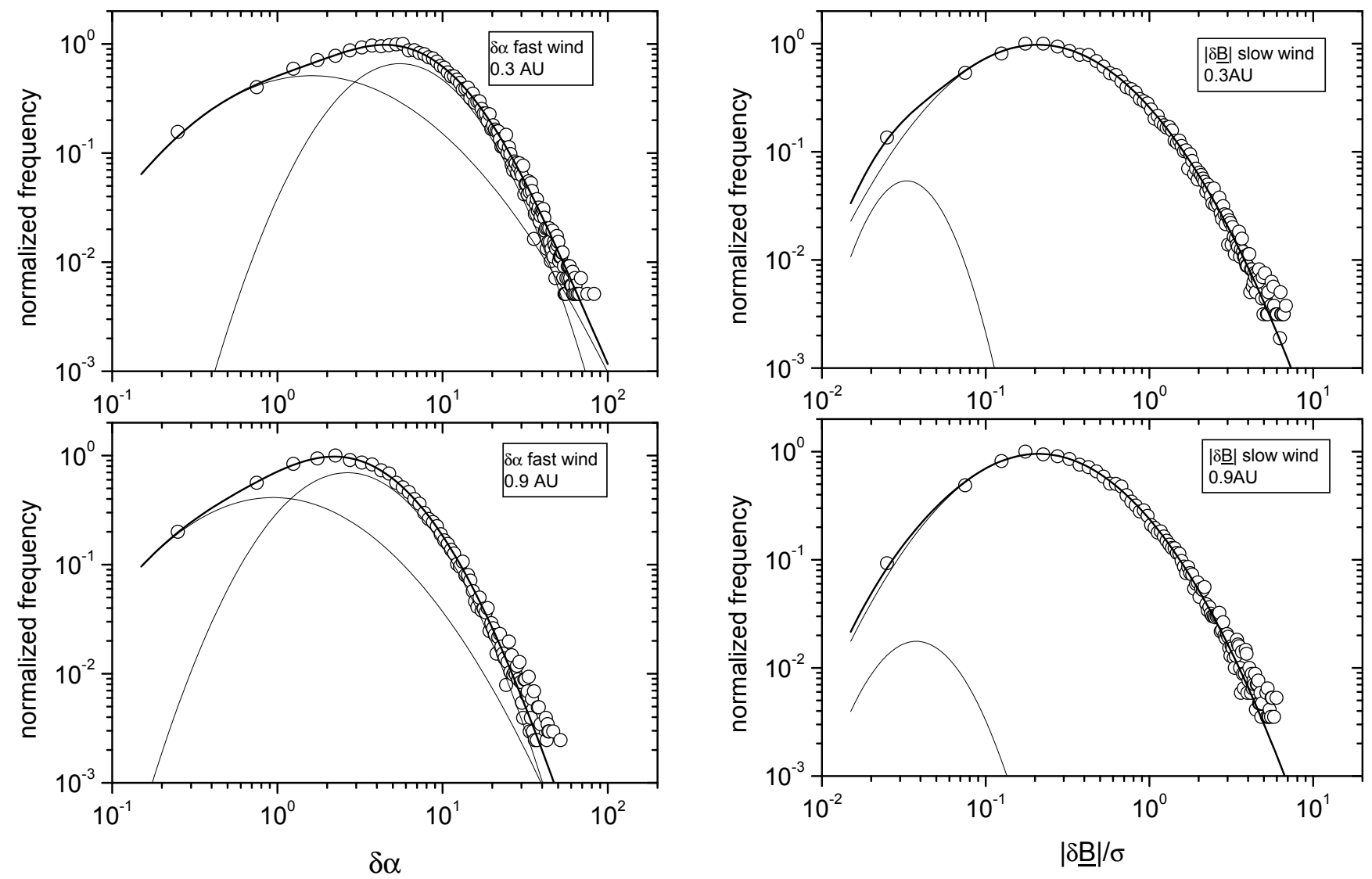

Fig. 9. PDFs of angular displacements $\delta \alpha$ at 0.3 and $0.9 \mathrm{AU}$, for a fast wind, are shown in the top and bottom panels, respectively. The two thin solid curves refer to as many lognormals contributing to form the thick solid curve which best fit the distribution. Parameters relative to the fit are reported in Table 3.

parameters inferred from the fit of the main lognormal, is remarkable which highlights the absence of radial evolution. In this case, values of $\delta$ are considerably smaller than those obtained for a fast wind, confirming that these fluctuations are generally smaller.

As already reported for a fast wind, we like to show the PDFs relative to the angular fluctuations as shown in Fig. 11, and parameters relative to the best fit, which are shown in Table 5. Also in this case, the contribution of the smaller lognormal is much smaller than within a fast wind. As a matter of fact, the ratio $\mathrm{A}_{2} / \mathrm{A}_{1}$ varies from $7.5 \%$ at $0.3 \mathrm{AU}$ to $4.4 \%$ at $0.9 \mathrm{AU}$. Moreover, these PDFs are roughly peaked at the same angle $\left(0.75^{\circ}\right)$, although the right tail at $0.3 \mathrm{AU}$ reaches larger values and does not show any noteworthy radial dependence.

\subsection{Building artificial interplanetary time series}

At this point, we tried to reproduce, from a statistical point of view, our interplanetary data samples by employing a random walk process governed by a double lognormal statistic acting on the direction of a unit vector. In other words, the

Fig. 10. PDFs of vector displacements $|\delta \underline{B}|$ normalized to $\sigma$ at 0.3 and $0.9 \mathrm{AU}$, for a slow wind, are shown in the top and bottom panels, respectively. The two thin solid curves refer to as many lognormals contributing to form the thick solid curve which best fits the distribution. Parameters relative to the fit are reported in Table 4. The smaller lognormal is almost superfluous since its contribution to the final fit is negligible.

interval of variability of $\left|\delta \underline{B}_{i}\right|$, as inferred from real data, was divided into a sufficient number of bins. For each of them we generated a certain number of values, all equal to the value represented by the mid point of the bin. The number of values generated depended on the corresponding probability indicated by the double lognormal, which was shaped using the same parameters that we had previously obtained from our best fits and reported in Tables 2 and 4 . These $\left|\delta \underline{B}_{i}\right|$ were then randomly extracted and used to make the tip of a unit vector, with one end fixed at the center of a sphere of unit radius and the other end free floating on the surface of the sphere. The direction of the path followed by the tip of the vector at each step was randomly extracted between $0^{\circ}$ and $360^{\circ}$. In particular, to avoid the effect of the two singular points at the poles of the sphere, the Cartesian coordinates of our reference system were rotated after each extraction in order to have the $x$ axis always coinciding with the newly extracted direction.

Four artificial temporal series of 28800 data points each, representing interplanetary observations performed at 0.3 

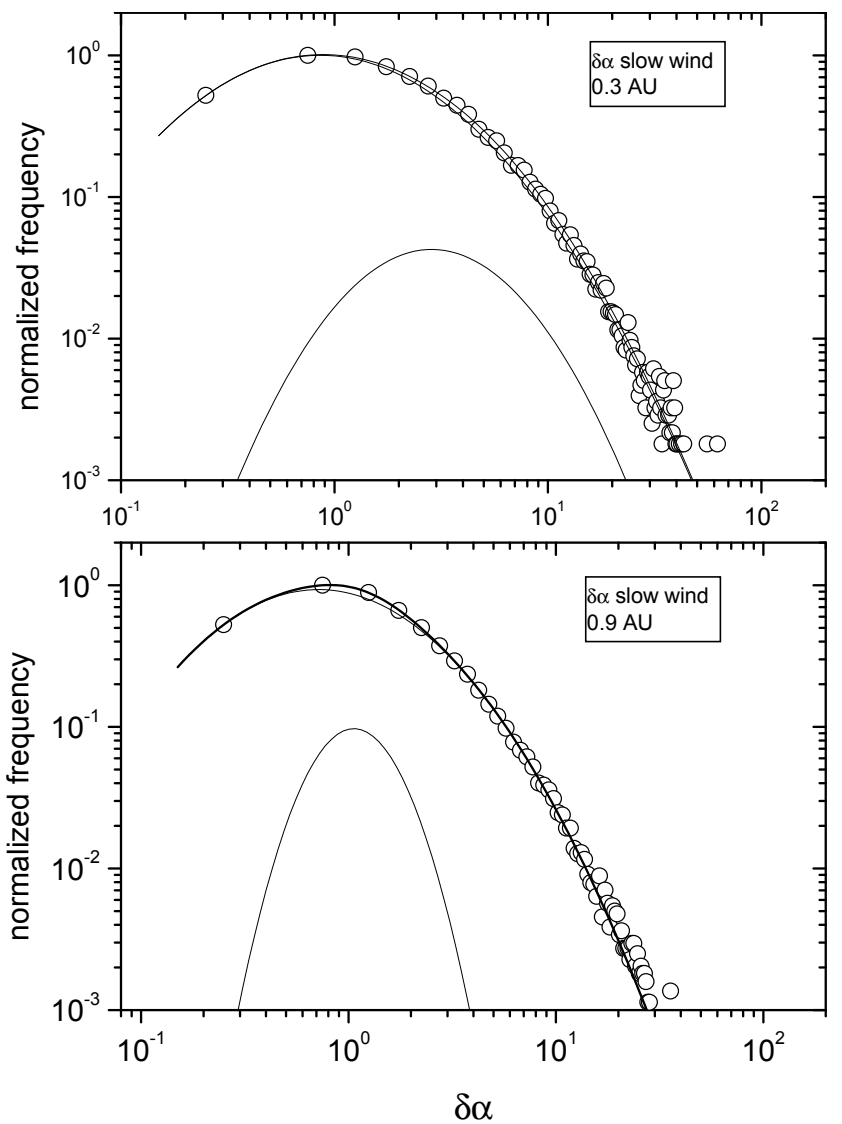

Fig. 11. PDFs of angular displacements $\delta \alpha$ at 0.3 and $0.9 \mathrm{AU}$, for a slow wind, are shown in the top and bottom panels, respectively. The two thin solid curves refer to as many lognormals contributing to form the thick solid curve which best fit the distribution. Parameters relative to the fit are reported in Table 5. Also in this case, the contribution of the smaller lognormal to the final fit is negligible.

and 0.9 AU, for a fast and a slow wind were built in such a way. Samples of 2000 data points each are plotted in Fig. 12 and Fig. 13, for a fast and a slow wind, respectively. The only arbitrary imposition we applied on the fast wind sample was that to always keep the same vector polarity to resemble, as much as possible, the real situation within a fast wind. As a consequence, we forced these fluctuations to remain within a solid angle of $2 \pi$ aperture. These plots reproduce at some level the main features that can be observed in Figs. 2 and 3. Fluctuations appear more intermittent in slow wind but show the largest evolution, between 0.3 and $0.9 \mathrm{AU}$, within a fast wind. Obviously, all the artificial time series we built have, by definition, the same statistics of real interplanetary data and we omit showing the PDFs of the relative $\left|\delta \underline{B}_{i}\right| / \sigma$ or $\delta \alpha$.

On the other hand, we like to show temporal sequences of these $|\delta \underline{B}(t)|$ relative to a fixed, arbitrary direction, as we did for real data shown in the top panels of Figs. 4 to 7 . Only the top panels have to be considered, since artificial data have been built to keep the vector intensity constant. Results are quite satisfactory since we are able to reproduce the typical behavior observed within both a fast and a slow wind.

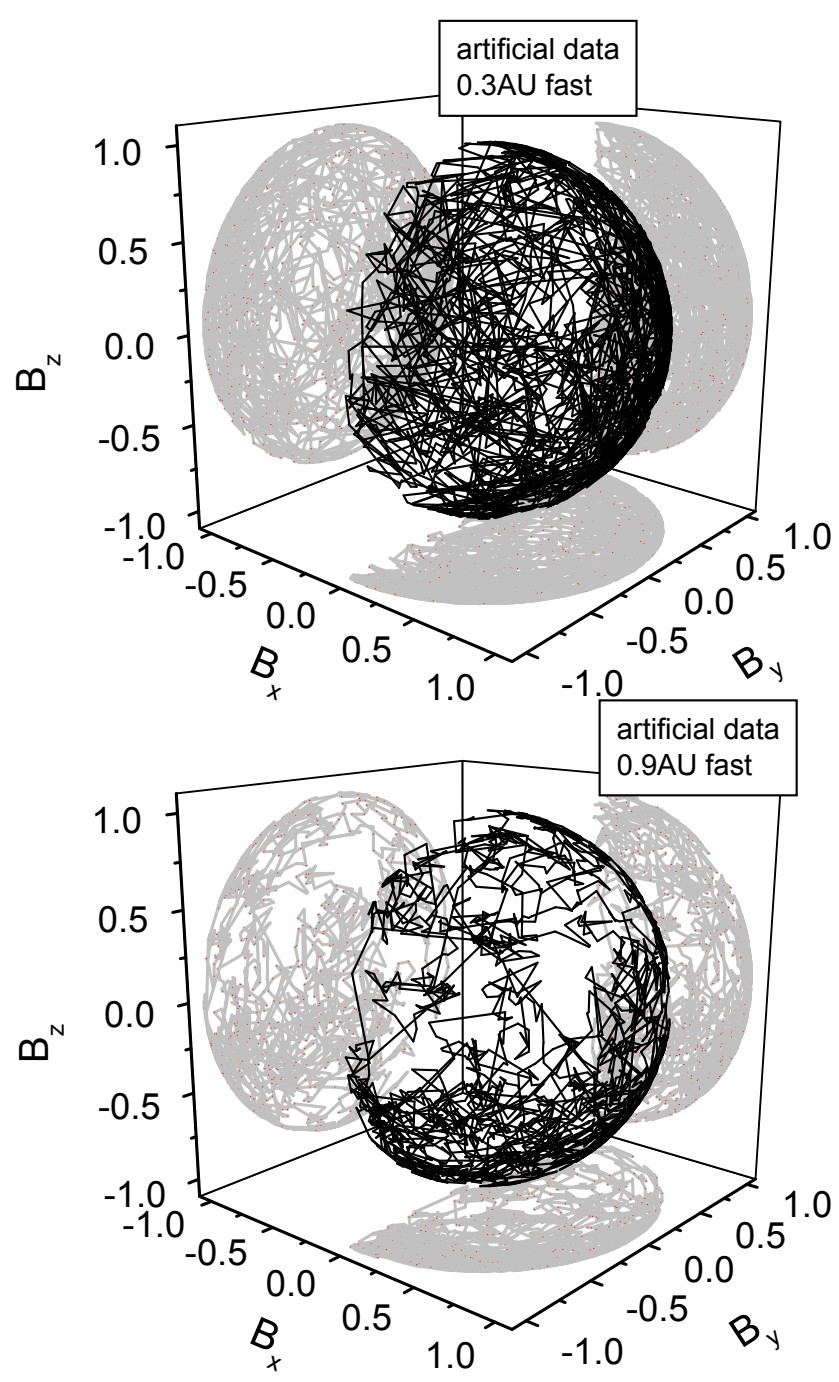

Fig. 12. Three-dimensional representation of vector displacements relative to artificial data generated by a random-walk, whose jumps obey a double-lognormal, whose parameters have been obtained by the best fit of real fluctuations. The top and bottom panels refer to fast wind at 0.9 and $0.3 \mathrm{AU}$, respectively, and have to be compared to analogous plots for real data shown in Fig. 2.

In particular, the transition from the chaotic behavior on the left panel of Fig. 14, representing fluctuations at $0.3 \mathrm{AU}$, towards more structured fluctuations on the right panel of the same figure, representing fluctuations at $0.9 \mathrm{AU}$, is well reproduced by the artificial time series.

Moreover, artificial data reproduce equally well fluctuations encountered at both heliocentric distances in a slow wind. They show similar structured fluctuations and not relevant differences between 0.3 and $0.9 \mathrm{AU}$.

The last comparison between the real and artificial time series that we like to show refers to the power spectra associated with fluctuations experienced by the vector components. In order to do so, we computed the trace of the power spectrum for real and artificial fluctuations relative to both heliocentric distances within a fast wind. Results are shown in 
Table 2. Fast Wind: Values of the parameters obtained from the fit of the PDF relative to vector displacements.

\begin{tabular}{cccccccc}
\hline Distance $\mathrm{AU}$ & $\mathrm{r}^{2}$ & $\mathrm{~A}_{1}$ & $\sigma_{1}$ & $\delta_{1}$ & $\mathrm{~A}_{2}$ & $\sigma_{2}$ & $\delta_{2}$ \\
\hline 0.3 & 0.998 & $0.85 \pm 0.13$ & $0.71 \pm 0.03$ & $0.95 \pm 0.02$ & $0.44 \pm 0.14$ & $1.08 \pm 0.04$ & $0.60 \pm 0.13$ \\
0.9 & 0.998 & $0.89 \pm 0.24$ & $0.74 \pm 0.03$ & $0.80 \pm 0.02$ & $0.20 \pm 0.25$ & $0.98 \pm 0.09$ & $0.52 \pm 0.32$ \\
\hline
\end{tabular}

Table 3. Fast Wind: Values of the parameters obtained from the fit of the PDF relative to angular fluctuations.

\begin{tabular}{cccccccc}
\hline Distance $\mathrm{AU}$ & $\mathrm{r}^{2}$ & $\mathrm{~A}_{1}$ & $\sigma_{1}$ & $\alpha_{1}$ & $\mathrm{~A}_{2}$ & $\sigma_{2}$ & $\alpha_{2}$ \\
\hline 0.3 & 0.998 & $8.47 \pm 0.76$ & $0.72 \pm 0.02$ & $9.24 \pm 0.14$ & $4.77 \pm 0.84$ & $1.16 \pm 0.03$ & $6.28 \pm 0.81$ \\
0.9 & 0.999 & $4.62 \pm 0.78$ & $0.75 \pm 0.03$ & $4.67 \pm 0.09$ & $1.87 \pm 0.82$ & $1.07 \pm 0.05$ & $3.00 \pm 0.82$ \\
\hline
\end{tabular}

Fig. 16, where power spectra relative to real fluctuations are reported in the left-hand panel while corresponding spectra of artificial time series are in the right-hand panel.

The power spectra of the components have been computed via a Fast Fourier Transform from time series of 2048 data points. The power spectral densities of the three components have been successively added up to obtain the trace of the spectral matrix which has been smoothed by averaging adjacent data points within a sliding window of 5 points. In the same panels we also show as a reference the slope of the classical Kolmogorov's spectrum. Spectra shown in the left-hand panel are typical spectra encountered within high velocity streams, as several times reported in literature (see review by Tu and Marsch, 1995). On the other hand, artificial spectra have been graphically separated by multiplying the spectrum identified by the label $0.3 \mathrm{AU}$ by a factor of $10^{2}$ to avoid overlapping. As a matter of fact, our artificial fluctuations have statistically similar amplitudes, no matter whether we refer to 0.3 or $0.9 \mathrm{AU}$, since our fluctuations are confined onto the surface of a sphere of unitary radius. Unexpectedly, the resemblance is so good that the artificial spectrum at $0.3 \mathrm{AU}$ shows a bending similar to the one that characterizes real fluctuations. The main differences seem to be in the high frequency tail of artificial data where effects due to aliasing, absent in the left-hand panel, can be noticed.

\section{Results of the numerical simulations of parametric in- stability}

Recently, Primavera et al. (1999); Malara et al. (2000, 2001); Primavera et al. (2003) investigated in detail how the parametric instability could be responsible for typical features observed in the radial evolution of the Alfvénic turbulence in the solar wind high speed streams. This instability develops in a compressible plasma and, in its simplest form, involves the decay of a large amplitude Alfvén wave (generally called a "pump wave", or "mother wave") in a magnetosonic fluctuation and a backscattered Alfvén wave. The wavevectors and frequencies of the fluctuations generated in this process are mutually related through well precise "resonance conditions" (Sagdeev and Galeev, 1969). This mechanism can be viewed as a way for decorrelating an initially coherent state (the large amplitude mother Alfvén wave). In fact, a circularly polarized Alfvén wave is an exact solution of the ideal magnetohydrodynamics equation, even in the compressible case. Hence, it should propagate unperturbed in a uniform plasma. However, in the presence of even very small perturbations in density, this wave is subject to the parametric instability and it decays, producing fluctuations of different kinds. In fact, in slow streams, the inhomogeneities of the background magnetic structure supply a source of decorrelation for the Alfvén waves coming from the sun (Bruno et al., 1985). On the converseside, in fast streams, where the magnetic field is more homogeneous, waves should travel almost undisturbed and the observed (although slower than in a slow wind) radial evolution of the Alfvénic turbulence need some mechanism to be ascribed to. Although it has been shown (Roberts et al., 1992; Goldstein et al., 1995) that plasma instabilities generated by velocity shears play a relevant role in the radial evolution of turbulence, another possibility is represented by parametric instability, as shown by Malara et al. (2000) and Primavera et al. (2003). These last studies related to parametric instability focus attention on the effects of this instability on the evolution of a large amplitude, circularly polarized, non-monochromatic Alfvén wave in a one-dimensional case. The spectrum of this initial perturbation has a break at a certain wavelength, like the spectrum of the Alfvénic fluctuations coming from the Sun. They found that the Alfvénic correlation of the initial perturbation is lost during the time evolution, because of the parametric instability, leading to a production of both backscattered Alfvénic perturbations and magnetosonic waves. Finally, these perturbations evolve nonlinearly, producing approximately power law spectra and a reduction in the normalized cross helicity. The results found are qualitatively in good agreement with solar wind observations carried out by several authors 
Table 4. Slow Wind: Values of the parameters obtained from the fit of the PDF relative to vector displacements.

\begin{tabular}{cccccccc}
\hline Distance $\mathrm{AU}$ & $\mathrm{r}^{2}$ & $\mathrm{~A}_{1}$ & $\sigma_{1}$ & $\delta_{1}$ & $\mathrm{~A}_{2}$ & $\sigma_{2}$ & $\delta_{2}$ \\
\hline 0.3 & 0.998 & $0.776 \pm 0.003$ & $0.959 \pm 0.009$ & $0.523 \pm 0.004$ & $0.002 \pm 0.001$ & $0.437 \pm 0.095$ & $0.039 \pm 0.010$ \\
0.9 & 0.998 & $0.727 \pm 0.004$ & $0.934 \pm 0.005$ & $0.503 \pm 0.004$ & $0.001 \pm 0.001$ & $0.532 \pm 0.722$ & $0.049 \pm 0.009$ \\
\hline
\end{tabular}

Table 5. Slow Wind: Values of the parameters obtained from the fit of the PDF relative to angular fluctuations.

\begin{tabular}{cccccccc}
\hline Distance $\mathrm{AU}$ & $\mathrm{r}^{2}$ & $\mathrm{~A}_{1}$ & $\sigma_{1}$ & $\alpha_{1}$ & $\mathrm{~A}_{2}$ & $\sigma_{2}$ & $\alpha_{2}$ \\
\hline 0.3 & 0.999 & $4.14 \pm 0.68$ & $1.08 \pm 0.02$ & $2.74 \pm 0.33$ & $0.31 \pm 0.66$ & $0.76 \pm 0.32$ & $5.12 \pm 0.76$ \\
0.9 & 0.999 & $2.68 \pm 0.02$ & $0.98 \pm 0.01$ & $1.89 \pm 0.01$ & $0.12 \pm 0.02$ & $0.42 \pm 0.03$ & $1.27 \pm 0.02$ \\
\hline
\end{tabular}

(see review by Tu and Marsch, 1995). In addition, Malara et al. (2000) observed that the turbulent development of the instability leads to the formation of shock waves and to an intermittent behaviour of the dissipation. In particular, looking at the evolution of the flatness of velocity and magnetic field fluctuations, Primavera et al. (2003) found a good qualitative agreement of the results of the simulations with the analysis of the same quantities performed by Bruno et al. (2003). It is then natural, in order to offer a possible different interpretation of the results shown in the previous sections, at least those concerning fast solar wind streams, to see whether turbulence induced by parametric instability has characteristics similar to those described in the solar wind in the previous sections. To accomplish this aim, we further analysed the results of the numerical simulations described in Primavera et al. (2003). The details of the numerical code can be found in Primavera et al. (1999), Malara et al. (2000) and Malara et al. (2001), whilst further details concerning the simulations are given in Primavera et al. (2003).

We simulate the evolution of a broad-band Alfvénic fluctuations in a compressible plasma, during their outward propagation in the heliosphere. Similar to the in-situ observations, the initial spectrum has a break point. During the run of the simulation, inward propagating fluctuations start to appear and form a power-law spectrum at small values of $\mathrm{k}$. As already pointed out by Tu et al. (1989), this feature might suggest that a parametric decay mechanism is at work in the solar wind.

The simulation domain is one-dimensional, periodic and we use Cartesian geometry.

The reference frame is chosen in such a way that the initial Alfvén wave is circularly polarized in the $x-z$ plane and it propagates along the $y$ direction. A background constant magnetic field intensity $B_{0}$ is imposed in the propagation direction of the wave: the resulting total field has, therefore, uniform intensity everywhere.

The homogeneous boundary conditions limit the application of this study to the fast wind, where the background magnetic field is rather homogeneous. In our framework, the time evolution of the quantities represents the radial evolution of the fluctuations in the solar wind, while the spatial variations are the numerical counterpart of samples of the observed data at a given distance from the sun. We study the evolution of the parametric instability for $180 \tau_{A}\left(\tau_{A}\right.$ is the Alfvén time based on the initial background radial magnetic field and density, i.e. the time needed for the wave, whose wavelength is the largest in our spectral domain, to go across the simulation box). In the rest of the paper, we plot quantities at time $t_{1}=45 \tau_{A}$ and $t_{2}=180 \tau_{A}$, the former corresponding to a time much before the saturation of the instability, the latter to a time longer than the saturation time, which is reached at $t_{\mathrm{sat}} \sim 100 \tau_{A}$. Practically, we consider a situation in which the instability has only weakly taken place and another in which it has already completely developed, that should be representative of the state of the solar wind closer to the Sun and further away from it, respectively.

We estimated the time needed by the instability to saturate and to reproduce the spectral features observed at $0.9 \mathrm{AU}$. We found that a period of time between 6 and 7 days is necessary. This estimation, although longer, is still within the same order of magnitude of the expansion time required by the solar wind to travel between 0.3 and $1 \mathrm{AU}$. The above evaluation is based on the fact that between $t_{1}$ and $t_{\text {sat }}$ there are about $55 \tau_{A}$. In order to estimate $\tau_{A}$ we compared the frequencies corresponding to the observed spectral break at $0.3 \mathrm{AU}$ in Helios data with the corresponding one shown in our simulation at $t=t_{1}$.

In Fig. 17, we show the three-dimensional plots of the magnetic field components, normalized to the average magnetic field intensity, at the time $t_{1}$ (upper panel) and $t_{2}$ (lower panel), respectively. These plots are to be compared to Fig. 2. One can see that at $t=45 \tau_{A}$ the tip of the magnetic field vector (represented by the line) moves in space in a rather uniform way and covers approximately the surface of a cylinder. This behaviour is qualitatively very similar to that shown in the upper panel of Fig. 2, except, in comparison with that 

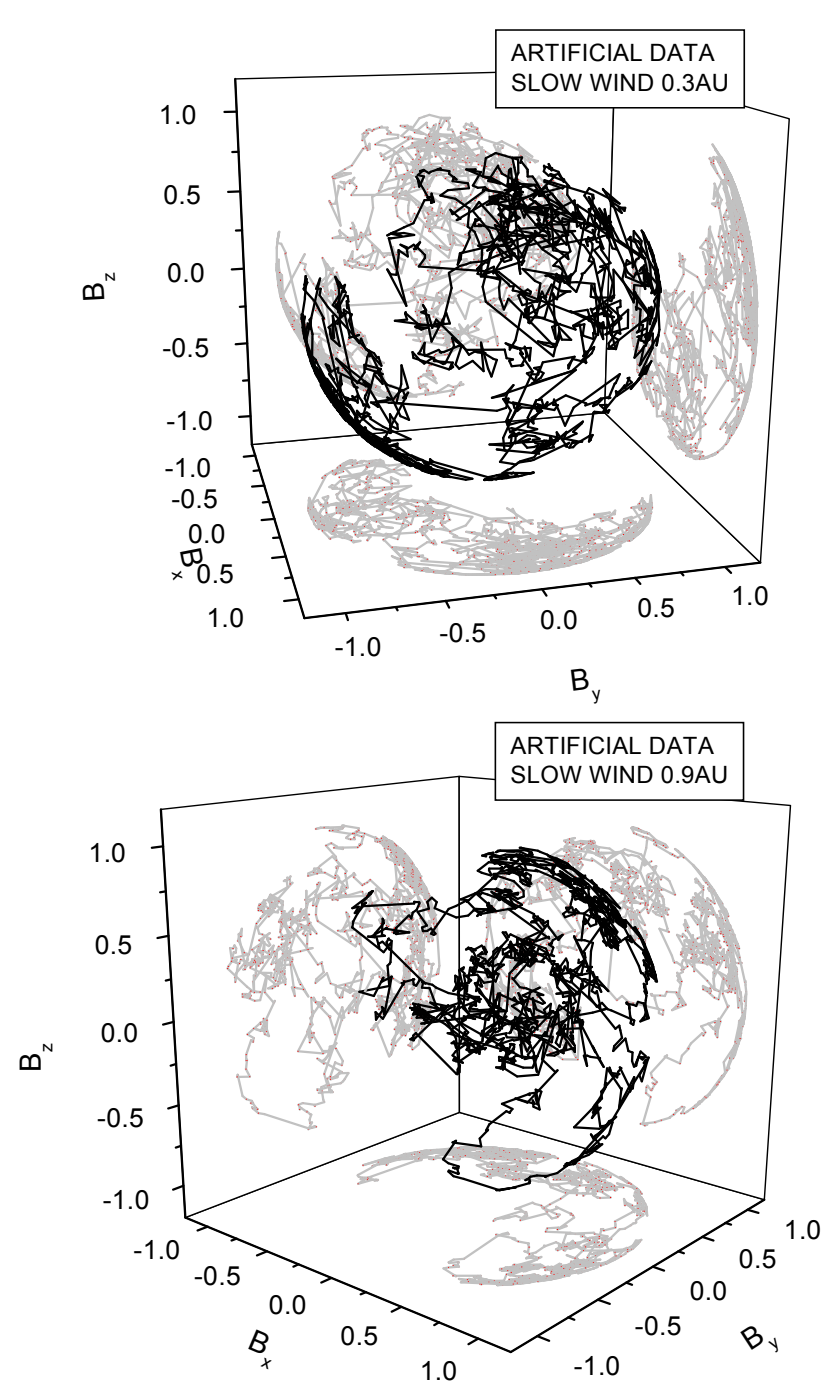

Fig. 13. Three-dimensional representation of vector displacements relative to artificial data generated by a random-walk, whose jumps obey a double-lognormal, whose parameters have been obtained by the best fit of real fluctuations. The top and bottom panels refer to a slow wind at 0.9 and $0.3 \mathrm{AU}$, respectively, and have to be compared to analogous plots for real data shown in Fig. 3.

case, here the tip of the vector covers almost uniformly a cylinder instead of a sphere. This difference is due to the fact that the numerical simulation is one-dimensional: this implies (due to the divergenceless condition for the magnetic field) that the variations of the magnetic field components are only orthogonal to the propagation direction of the waves, whilst the parallel component remains constant during the time evolution. Moreover, since the initial perturbation is circularly polarized, the trajectory of the tip of the vector would describe a circular line. In order to improve the visualization of the curve, to make it three-dimensional instead of two-dimensional, we replaced the constant component of the magnetic field with a linearly growing function between zero and one, that makes the tip of the vector stay on a cylindrical surface. The real data are three-dimensional instead,

and the approximately constant field intensity produces the spherical pattern plotted in Fig. 2, as already explained in Sect. 2.

At the later time, $t_{2}=180 \tau_{A}$, the situation changes dramatically. The tip of the magnetic field vector describes a more patchy pattern, characterized by large jumps, followed by smaller fluctuations around a single direction, and so on. This pattern is qualitatively similar to that observed in Fig. 2. In conclusion, results of parametric instability simulation seem to account for the observed transition to a sort of Lévy walk observed in the real solar wind data (see also Bruno et al. (2004)).

As a second point of agreement with the observations, we plot the vector displacements $|\delta \underline{B}|$, with respect to a fixed direction, normalized to the mean magnetic field intensity as a function of the independent variable $y$. We compute this quantity by considering the magnetic field at a given time $t$ and evaluating the vector differences of its components with the fixed direction $(0 ; 1 ; 0)$ in each simulation grid point. Since in our simulation the background magnetic field has components $\underline{\boldsymbol{B}}_{\mathbf{0}}=(0 ; 1 ; 0)$, we are practically plotting the vector displacements of the magnetic field fluctuations. Finally, we compute the intensity of this vector in each point. The results of this computation are shown in the upper panel of Fig. 18 at the time $t_{1}=45 \tau_{A}$ and Fig. 19 at the time $t=180 \tau_{A}$. Along with these curves, the magnetic field intensities $\underline{\mid \boldsymbol{B}}(\boldsymbol{y}) \mid$, normalized to its mean value, at the same times, are shown in the lower panels of the figures. These graphics should be compared with the ones in Figs. 6 and 7, where the analogous quantities are plotted for the fast solar wind at 0.3 and $0.9 \mathrm{AU}$, respectively.

Also in this case, the qualitative similarity between the results of the simulations and the observed solar wind data is remarkable. At the time $t=t_{1}$, corresponding to $0.3 \mathrm{AU}$, the vector displacements have a quite random behaviour, although not as "noisy" as in Fig. 6, and no evident correlation between the vector displacements and the magnetic field intensity is observed. Note that the fluctuations of the magnetic field intensity are rather small at this time, due to the fact that the initial wave is circularly polarized.

On the converseside, in Fig. 19, the vector displacements of the magnetic field appear to be more structured, characterized by fast rotations of the vector, followed by smaller oscillations around the new position. Moreover, there exists a clear correlation between the strongest gradients of the vector displacements and the ones of the magnetic field intensity, as already observed in the real solar wind fast streams.

Finally, we plotted in Fig. 20 the PDFs of vector displacements $\left|\delta \underline{B}_{\lambda}\right|$, defined as

$\left|\delta \underline{B}_{\lambda}(x, t)\right|=\sqrt{\sum_{i=x, y, z}\left(B_{i}(x+\lambda, t)-B_{i}(x, t)\right)^{2}}$,

normalized to their standard deviations, at times $t_{1}$ and $t_{2}$. In Eq. (3), $t$ represents a specific phase of the evolution, $x$ a generic position within the simulation box and $\lambda$ is the scale length analogous to the sampling time of real data used to 

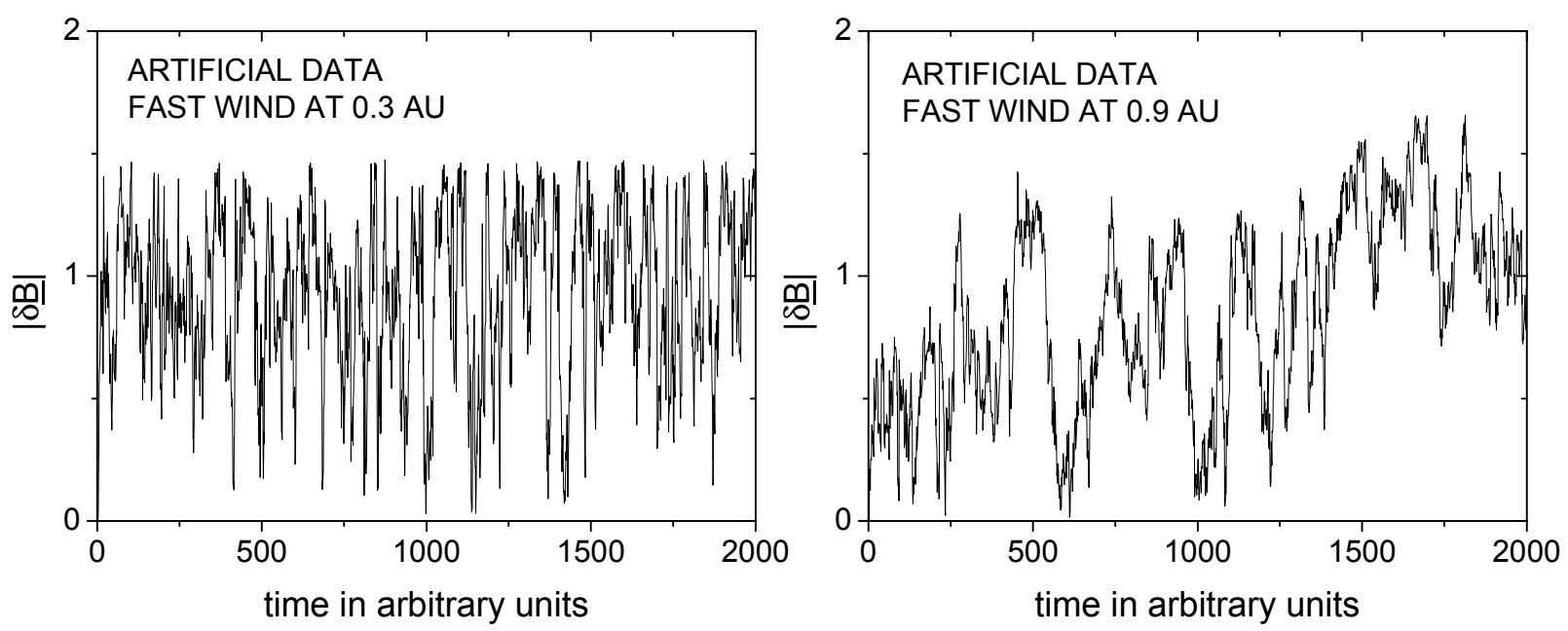

Fig. 14. Vector displacement versus time as measured from artificial data referring to a fast wind at $0.3 \mathrm{AU}$ on the left panel and at $0.9 \mathrm{AU}$ on the right panel. These plots should be compared to the top panels of Figs. 6 and 7, respectively.
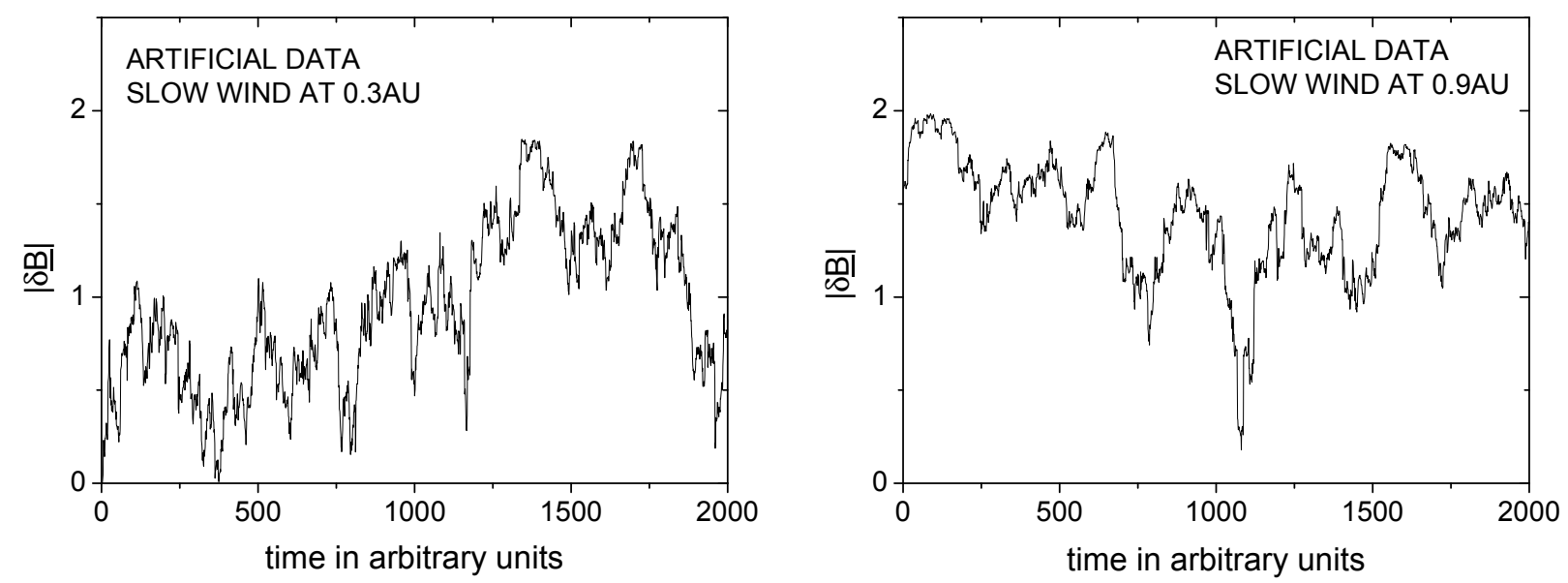

Fig. 15. Vector displacement versus time as measured from artificial data referring to a slow wind at 0.3 AU on the left panel and at 0.9 AU on the right panel. These plots should be compared to the top panels of Figs. 4 and 5, respectively.

compute the PDFs in Sect. 2. However, in comparison with real data, where the resolution of $6 \mathrm{~s}$ still lies in the inertial range, in the simulations we have to take into account the fact that the smaller scales are affected by viscosity and diffusivity. Thus, we evaluated the vector differences at a length scale $\lambda=\lambda_{\max } / 628$, where $\lambda_{\max }$ is the maximum wavelength excited at the beginning of the simulation, and equals the length of the simulation domain. This length scale is rather small, compared to the integral scale of the domain, but still in the inertial range of the spectrum.

The similarity with Fig. 8, where the PDFs of the vector displacements $|\delta \underline{B}|$ are shown for the fast wind at 0.3 and $0.9 \mathrm{AU}$, is evident. Also in this case, it is possible to fit effectively the curves with two lognormal distributions and the trend is similar to that observed in the solar wind data. In fact, the two lognormal distributions have comparable heights at $\mathrm{t}=\mathrm{t}_{1}$, while the population relative to the long vector displacements increases its importance at subsequent times $\left(\mathrm{t}=\mathrm{t}_{2}\right)$.
The parameters of the fits are shown in Table 6 .

Another point of similarity with the analysis of real data regards the power spectrum. As a matter of fact, as already shown by Malara et al. (2000) and Malara et al. (2001), the power spectrum obtained from the trace of the spectral matrix of the Alfvénic fluctuations, after the saturation of the parametric instability has been reached, shows clear evidence of a power-law inertial range similar to the one observed in the Helios data at $0.9 \mathrm{AU}$.

We like to stress that a direct quantitative comparison between the real data and the simulated data is meaningless. In fact, a direct comparison would require one to fix the appropriate length and time scales in the simulation. Moreover, as described in Primavera et al. (2003), the model used is very simple, due to the one-dimensionality constraint, to the Cartesian geometry and especially to the fact that the solar wind is actually an expanding medium. However, the results of the simulations show a qualitative behaviour very similar 

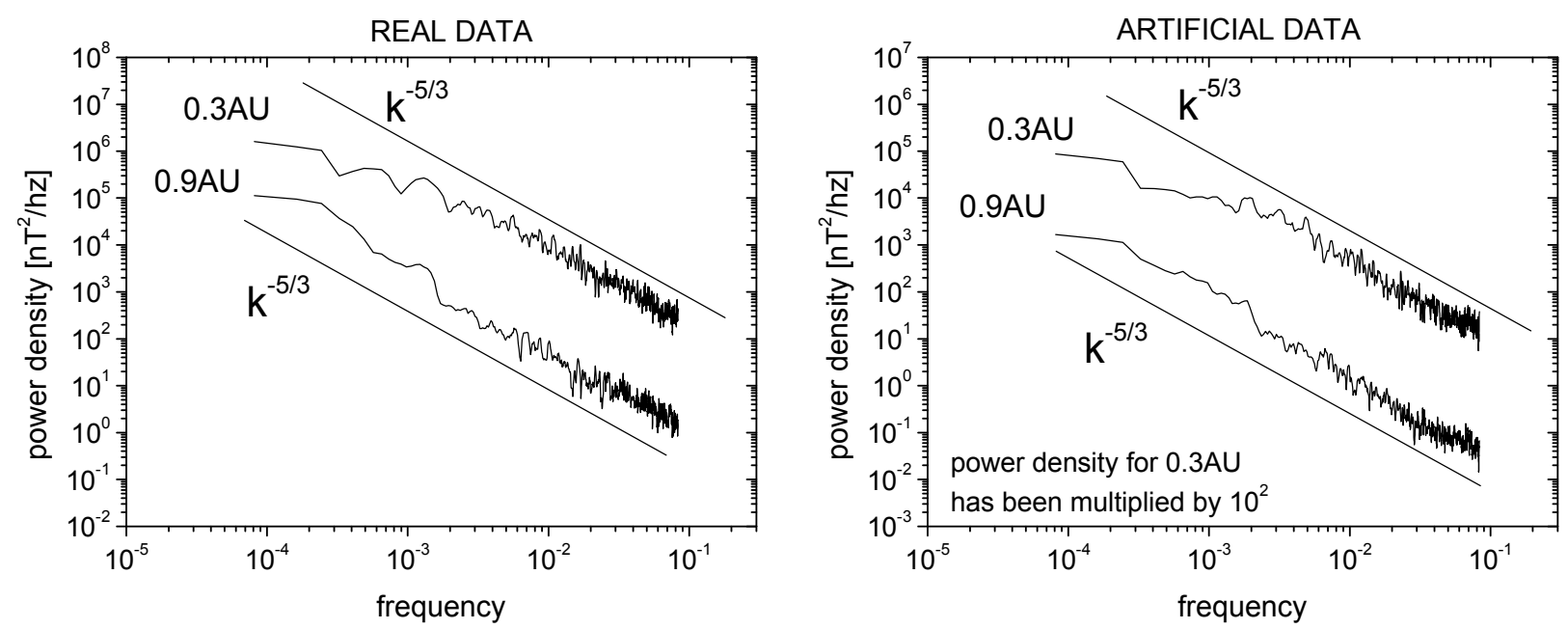

Fig. 16. The left panel shows power spectra obtained from the trace of the spectral matrix relative to magnetic field fluctuations recorded at 0.3 and 0.9 AU by Helios 2 in 1976. Each time interval had 2048 averages of $6 \mathrm{~s}$ each and are the same sub-intervals used for Figs. $2,6,7$. The right panel shows power spectra obtained from the trace of the spectral matrix relative to the artificial field fluctuations relative to 0.3 and $0.9 \mathrm{AU}$, built from a random walk whose jumps obey a double-lognormal distribution. Data relative to $0.3 \mathrm{AU}$ have been multiplied by a factor of $10^{2}$ to facilitate visual comparison with real data shown in the left panel. It is worth noticing that artificial data, besides a general agreement with real data, are able to reproduce the bending of the power spectrum observed at $0.3 \mathrm{AU}$. Straight solid lines indicate the $\mathrm{k}^{-5 / 3}$ Kolmogorov slope.

Table 6. Numerical simulation: Values of the parameters obtained from the fit of the PDF relative to vector displacements.

\begin{tabular}{cccccccc}
\hline Simulated time & $\mathrm{r}^{2}$ & $\mathrm{~A}_{1}$ & $\sigma_{1}$ & $\delta_{1}$ & $\mathrm{~A}_{2}$ & $\sigma_{2}$ & $\delta_{2}$ \\
\hline $45 \tau_{A}$ & 0.954 & $1.13 \pm 0.14$ & $1.43 \pm 0.09$ & $1.08 \pm 0.20$ & $0.47 \pm 0.06$ & $0.35 \pm 0.03$ & $0.94 \pm 0.03$ \\
$180 \tau_{A}$ & 0.996 & $0.52 \pm 0.56$ & $0.72 \pm 0.21$ & $0.45 \pm 0.27$ & $0.09 \pm 0.55$ & $0.69 \pm 0.95$ & $0.15 \pm 0.45$ \\
\hline
\end{tabular}

to that observed in the real data of the fast solar wind. We conclude that the parametric instability offers a possible alternative explanation of the observed data.

\section{Conclusions}

In this paper we focused on the statistics followed by interplanetary magnetic field fluctuations on a 6-s time scale, well inside the MHD regime (Bavassano et al., 1982), as observed in solar wind turbulence between 0.3 and 0.9 AU. In particular, we aimed to understand the spatio-temporal evolution of the magnetic field vector through the study of changes experienced by both vector orientation and intensity. Several previous works, which dealt with a statistical approach to this same problem, considered different aspects connected to directional fluctuations as, for example, power associated with the fluctuations, their radial evolution, their anisotropy, the nature of the fluctuations, their generation mechanisms, and so on, but none of them, to our knowledge, has ever studied how and why the orientation of these fluctuations changes with time. There have been only a few attempts to study similar problems but always limited to single case studies (Nakagawa et al., 1989; Tu and Marsch, 1991; Tsurutani et al., 1994; Riley et al., 1996; Bruno et al., 2001). The most recent statistical approach to the same problem is represented in a paper by Bruno et al. (2004), in which these authors concluded that the temporal evolution of the magnetic field and wind velocity vectors directions might follow a sort of Lévy walk. That paper, although based on larger time scales and on weak statistics, represents the first attempt to understand the influence due to propagating modes and convected structures on the orientation of velocity and magnetic field vectors within MHD turbulence. Following this analysis and, using a more robust statistics, we found that PDFs of interplanetary magnetic field vector differences within high velocity streams can be reasonably fitted by a double lognormal distribution. In other words, vector differences, which are due to the two distinct contributions of directional uncompressive fluctuations and purely compressive fluctuations, can be separated into two distinct PDFs. Moreover, the lognormal nature of the PDFs might suggest a multiplicative process at the origin of these fluctuations, that is typical of a turbulent cascade.

Furthermore, it only applies to definite positive quantities, like the vector or angular displacements we analyze in this paper. 


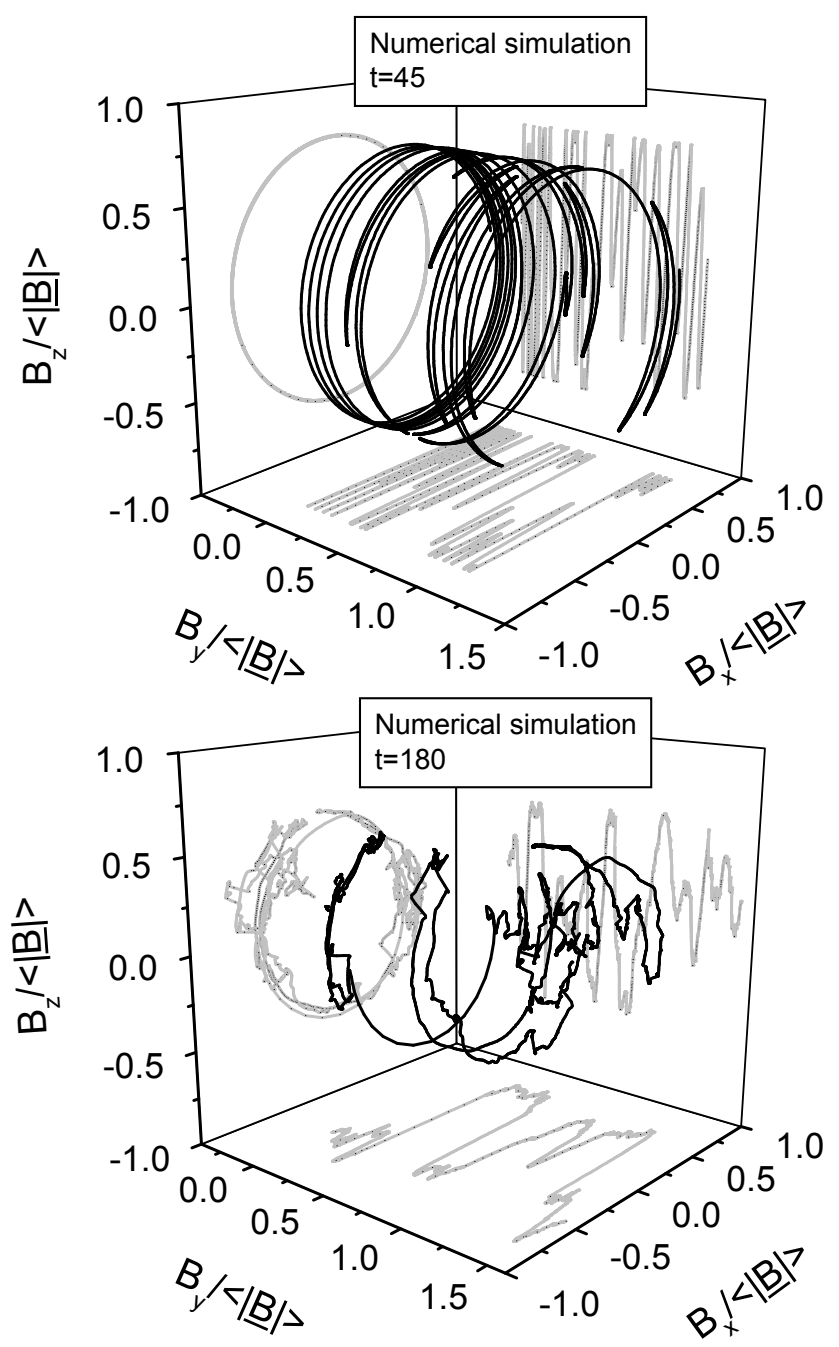

Fig. 17. The top panel of the figure refers to the results of the numerical simulations of parametric instability at time $t=t_{1}=45 \tau_{A}$, while the bottom panel refers to the the results at time $t=t_{2}=180 \tau_{A}$. Each point of both plots represents the location of the tip of the magnetic field vector in a point of the simulation domain, in the same format of Fig. 2, which the present figure should be compared with. The difference in the shape of the plots, cylindrical for this figure, spherical for Fig. 2, is explained in the text. Also in this case, the simulated data at $t=t_{1}$, corresponding to the real data at $0.3 \mathrm{AU}$, have a more uniform distribution on the cylindrical surface, whilst the data at $t=t_{2}$ (to compare with real data at $0.9 \mathrm{AU}$ ) show long jumps, followed by small amplitude oscillations around the new values.

Incidentally, the multiplicative cascade notion was introduced by Kolmogorov into his statistical theory (Kolmogorov, 1941, 1962) of turbulence as a phenomenological framework to accomodate extreme behaviour observed in real turbulent fluids.

Another interesting feature of these distributions is that the two PDFs have a different weight since one of them, the one that represents the smallest $\left|\delta \underline{B}_{i}\right|$ is always considerably smaller than the other one. Moreover, while the smaller PDF does evolve with heliocentric distance, decreasing its
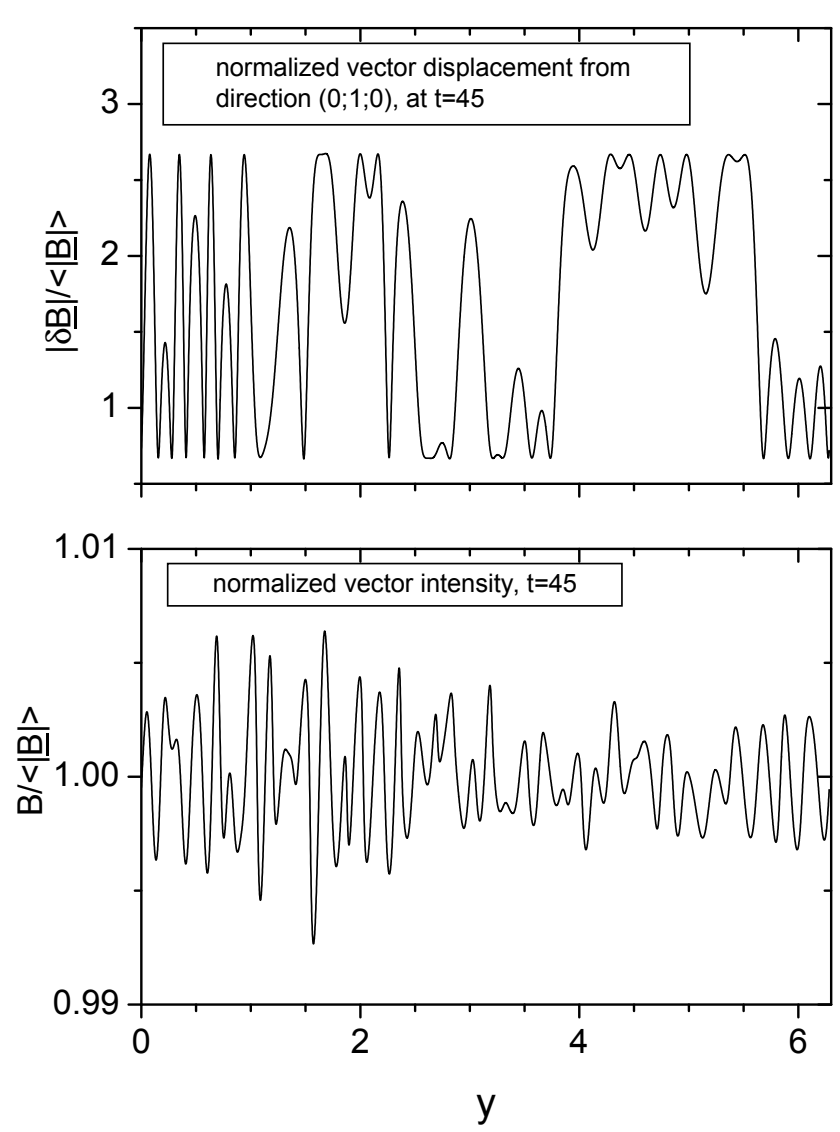

Fig. 18. Results of the numerical simulations of the parametric instability at time $t=t_{1}=45 \tau_{A}$. Normalized vector displacements versus the spatial coordinate $y$ are shown in the top panel, while normalized vector intensities are shown in the bottom panel. This figure should be compared with Fig. 6. The top panel shows large fluctuations which are basically uncorrelated with the profile of the magnetic field intensity in the bottom panel. This behaviour is qualitatively similar to that observed in the fast wind at $0.3 \mathrm{AU}$ (see Fig. 6)

own relevance, the largest PDF seems to remain almost unaffected. Now if we consider, as already suggested (Bruno et al., 2003), MHD turbulence mainly due to propagating, uncompressive fluctuations of Alfvénic origin and due to convected compressive structures, it comes naturally to identify these different contributions to turbulence with the two PDFs we found. In addition, we would expect a different radial evolution since only propagating Alfvénic modes, interacting nonlinearly, undergo a considerable turbulent evolution as we already know from literature (Tu and Marsch, 1995). We found that the relative contribution to the total PDF of what we identify with uncompressive fluctuations varies from $34 \%$ at $0.3 \mathrm{AU}$ to $18 \%$ to $0.9 \mathrm{AU}$, in terms of relative probability. In other words, what we identify with the Alfvénic contribution results to be somewhat smaller than the contribution due to the convected structures. Similar conclusions were reached by Bieber et al. (1996), who gave an estimate for the Alfvénic component around 15\% of the to- 

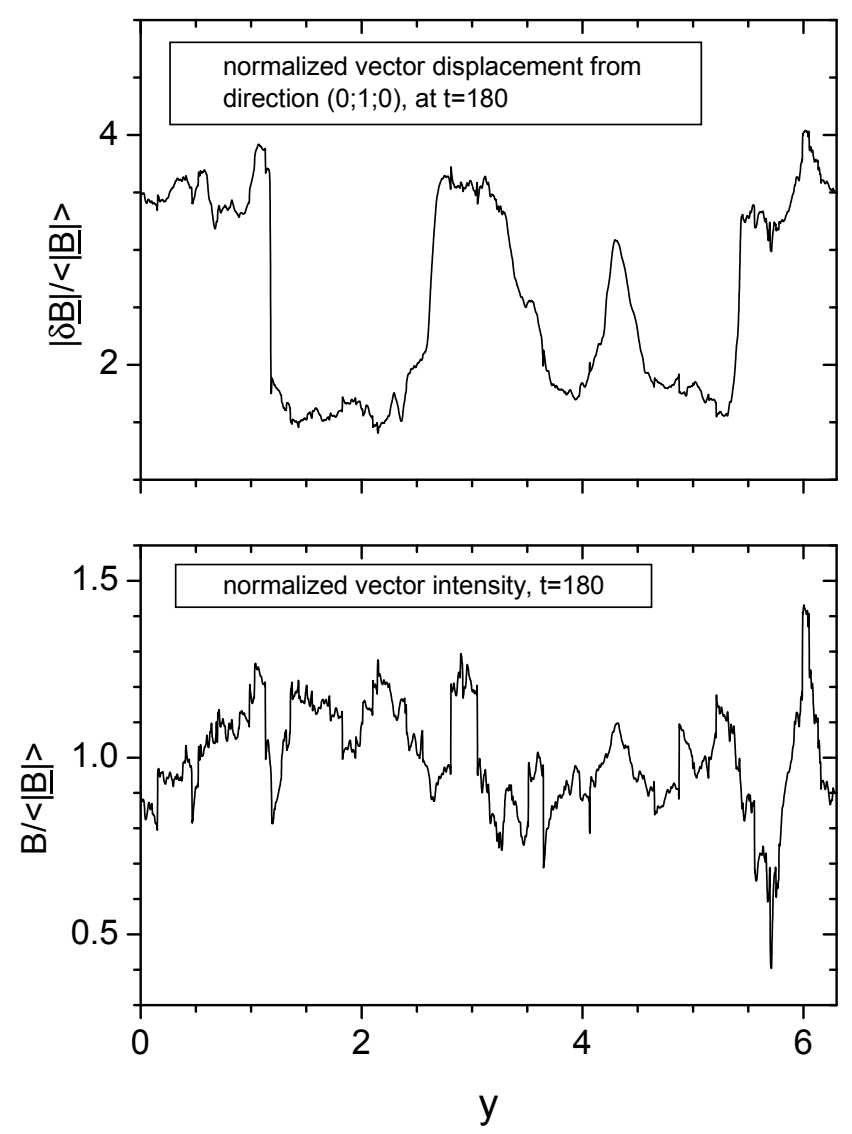

Fig. 19. Normalized vector displacements and normalized vector intensity versus position in the simulation box are shown in the same format as Fig. 18 for the results of the numerical simulations at time $t=t_{2}=180 \tau_{A}$. A difference with the previous figure, vector displacements (top panel) appear to be more structured in space and some sort of correlation with the field intensity (bottom panel) can be recognized. This behaviour is qualitatively similar to that observed in fast solar wind at 0.9 AU (Fig. 7).

tal power associated with turbulence. Thus, we might associate our convected structures to the 2-D turbulence identified in the solar wind by Bieber et al. (1996) and Matthaeus et al. (1990), who modeled interplanetary magnetic turbulence made of slab and quasi-2-D turbulence only. However, the dominant 2-D magnetic turbulence is characterized by the fact that its wave vector results to be normal to the ambient magnetic field direction. As a consequence, we would expect to see a radial evolution even stronger than the one we observed for the slab component which has its wave vectors parallel to the ambient field. As a matter of fact, the turbulent cascade acts preferably on wave numbers perpendicular to the ambient magnetic field direction, as suggested by the three-wave resonant interaction (Shebalin et al., 1983; Bondeson , 1985). On the contrary, the dominant component of the turbulence observed by Helios is the least affected by the radial evolution and probably should not be identified with the 2-D turbulence. Another possibility is that the 2-D turbulence is mixed together with the slab turbulence and is represented by the smaller PDF which experiences the stronger
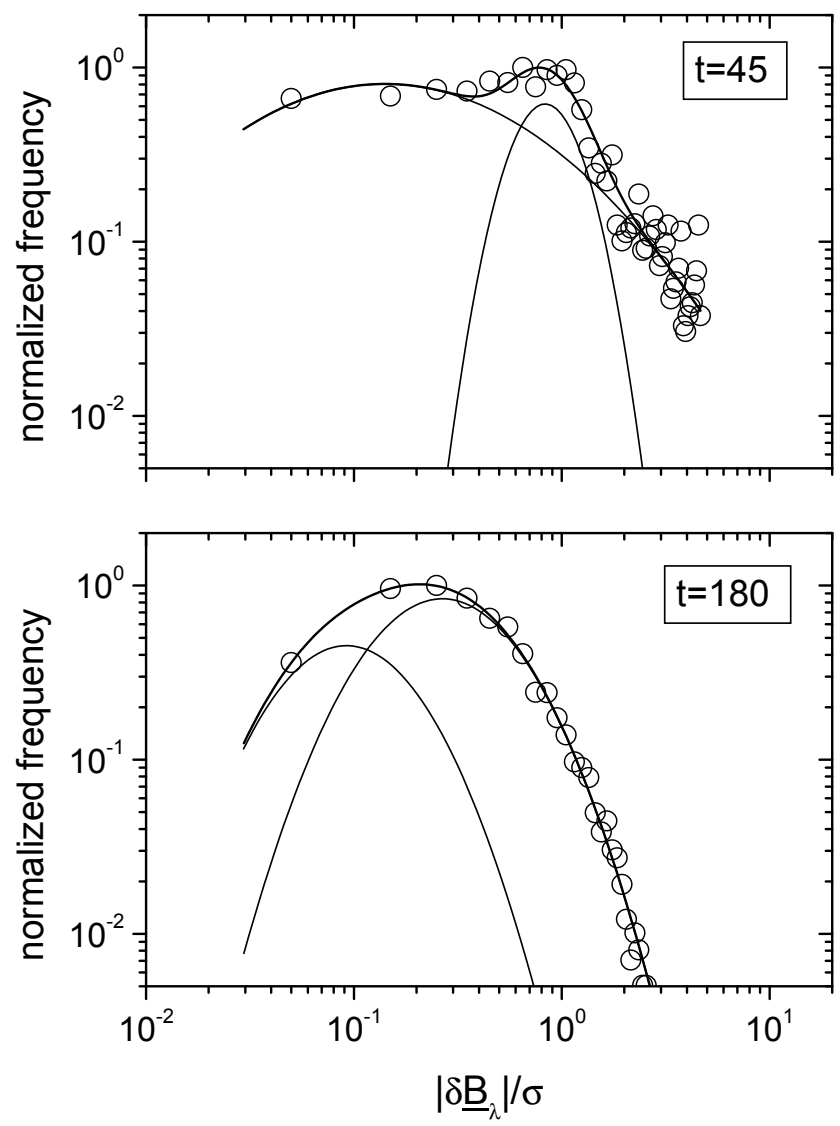

Fig. 20. PDFs of vector displacements $\left|\delta \underline{B}_{\lambda}\right|$, at the scale $\lambda=\lambda_{\max } / 628$, normalized to $\sigma$ for the results of the numerical simulations of the parametric instability at time $t=t_{1}=45 \tau_{A}$ and $t=t_{2}=180 \tau_{A}$ are shown in the top and bottom panels, respectively. The two thin solid curves refer to as many lognormals contributing to form the thick solid curve which best fits the distribution. Parameters relative to the fit are reported in Table 6.

radial evolution. If this is the case, our analysis suggests that interplanetary fluctuations are made of three rather than two components: slab, 2-D and convected structures which would support the three-component model by Matthaeus and Ghosh (1999). This view is corroborated by the fact that the PDF of $\left|\delta \underline{B}_{i}\right|$ within a slow wind can be fitted by a single lognormal whose parameters only slightly change with heliocentric distance. As a matter of fact, this behavior has to be expected if we consider that a slow wind is poor in Alfvén modes and its turbulence is already fully developed by the time we observe it at 0.3 AU (Tu and Marsch, 1990). Consequently, between .3 and $0.9 \mathrm{AU}$, fluctuations do not undergo the same turbulent evolution, observed in the fast wind and the constancy of the PDF of $\left|\delta \underline{B}_{i}\right|$ should be expected. We like to stress that interplanetary observations revealed that slow wind MHD fluctuations are intrinsically different from those observed in a fast wind. In fact, Bavassano et al. (2000) showed that the Elsässer ratio $\mathrm{e}^{-} / \mathrm{e}^{+}$, which is always around 1 within a slow wind, saturates to $\sim 0.5$ within a fast wind at a distance of $\sim 2.5 \mathrm{AU}$. 
One more interesting observation regards the topology showed by these fluctuations within a fast and a slow wind. We showed that the trajectory, followed by the tip of the magnetic vector during its turbulent fluctuations, follows a structured path. This path appears more clearly when the PDF of $\left|\delta \underline{B}_{i}\right|$ can be fitted by a single lognormal, as in the case of slow wind, regardless of heliocentric distance. However, within a fast wind this structured path can be more easily observed by increasing the heliocentric distance, in concurrence with the depletion of the Alfvénic fluctuations. In other words, Alfvénic modes mask the underlying magnetic, quasi-static structure convected by the wind. The superposition of these two types of fluctuations is such that the final motion is characterized by extreme behaviour. Referring to the 3-D representation used in this paper, the tip of the vector appears to be trapped within a certain solid angle for some time but occasionally it escapes this limited angular region and quickly travels, in a few time steps, to finally end up in another angular region characterized by a different average orientation. These large jumps should be accounted for by the larger PDF and should be related to similar large jumps studied by Bruno et al. (2001) and interpreted as tangential discontinuities marking the border between adjacent flux tubes. On the contrary, local fluctuations, clustering around certain average directions, should have an Alfvénic nature and should be identified by the smaller PDF. These results support and further corroborate the recently re-proposed spaghetti-like structure model (Bruno et al., 2001) first introduced, although in the context of cosmic ray modulation, by McCracken and Ness (1966), to describe interplanetary magnetic field topology.

Finally, adopting a sort of feedback procedure, we crosschecked the soundness of our fitting scheme, showing that artificial data obtained from the tip of a vector that randomly walks on the surface of a sphere of a constant radius, performing directional jumps, which obey a double lognormal, provides results similar, in some aspects, to those observed in interplanetary space.

However, the interplanetary observations we have do not allow one to understand whether these structures come directly from the Sun or are locally generated by some mechanism. Recent theoretical results by Primavera et al. (2003) showed that coherent structures responsible for the radial dependence of Intermittency, as observed in the solar wind (Bruno et al., 2003), might be locally created by the parametric decay of Alfvén waves. These authors showed that during the turbulent evolution, coherent structures, like shocklets and/or current sheets, were continuously created when the instability was active.

In order to see whether a similar mechanism may account for the observed behaviour of the vector displacements and their statistics, we further analyzed in this paper the results of the simulations performed in Primavera et al. (2003). The results of this investigation show a fairly good agreement, at least under the qualitative point of view, between the simulations and the solar wind data: either the evolution of the tip of the magnetic field vector, or the correlation between the vector displacement at a given scale with the magnetic field intensity fluctuations, or the evolution of the PDFs of the vector displacement in time, all show trends similar to those observed in the real fast solar wind data. Unfortunately, a direct quantitative comparison between the simulations and the data is difficult due to the limitations of the model.

However, this mechanism, which might be active within a fast wind, should be less effective within a slow wind, given the remarkable decoupling between the magnetic field and the velocity field within this type of wind (Klein et al., 1993).

Nevertheless, the enticing nature of the parametric instability in explaining the results comes from some well-defined fact: a) it is a well-defined mechanism of physical origin that induces a turbulent evolution in the plasma and not an undefined turbulence model; b) it is likely applicable to explain many general observed features of a fast solar wind, like the evolution of the spectra, the decrease of the Alfvénic correlation during the propagation in the heliosphere, and so on (Primavera et al., 2003); c) the observed evolution of the vector displacements and of their relative PDFs can be seen as a natural consequence of the formation of shocklets and discontinuities in the wind, organized in a sort of coherent structure, that explain the long jumps observed in the magnetic field and the structures in the vector displacements at larger distances from the Sun. In particular, the decrease with distance of the lognormal component of the PDFs correlated to the Alfvénic part of the turbulence, can be seen as the continuous transfer of energy between the Alfvénic and magnetosonic components of the waves during the evolution of the instability. However, a definitive conclusion about this point needs further investigation.

Another recent theoretical effort by Chang et al. (2004) models MHD turbulence in a way that tends to the view and interpretation of the interplanetary observations we presented in this paper, that is the existence of two different components both contributing to turbulence. The theoretical model presented by these authors tells us that propagating modes and coherent, convected structures are both necessary, inseparable ingredients of MHD turbulence, since they share a common origin within the general view described by the physics of complexity Chang (1999); Vasquez and Hollweg (2004); Vasquez et al. (2004). Propagating modes experience resonances which generate coherent structures which, in turn, will migrate, interact and eventually generate new modes.

These theoretical models, which favour the local generation of coherent structures, fully complement the possible solar origin of the convected component of interplanetary MHD turbulence.

Acknowledgements. Magnetic field $6 \mathrm{~s}$ averages derive from the Rome-GSFC magnetic experiment onboard Helios $2 \mathrm{~s} / \mathrm{c}$. (PIs of the experiment were $F$. Mariani and N. F. Ness)

Topical Editor R. Forsyth thanks S. Oughton and C. Tu for their help in evaluating this paper. 


\section{References}

Anselmet, F., Gagne, Y., Hopfinger, E. J., and Antonia, R. A.: High order velocity structure functions in turbulent shear flows, J. Fluid Mech., 140, 63-89, 1984.

Bavassano, B., Dobrowolny, M., Mariani, F., and Ness, N. F.: Radial evolution of power spectra of interplanetary Alfvenic turbulence, J. Geophys. Res. 86, 3617-3622, 1982.

Bavassano, B., Pietropaolo, E., Bruno, R.: On the evolution of outward and inward Alfvnic fluctuations in the polar wind, J. Geophys. Res., 105, 15 959-15 964, 2000.

Belcher, J. W. and Davis, L. Jr.: Large-amplitude Alfvén waves in the interplanetary medium, J. Geophys. Res. 76, 3534-3563, 1971.

Bieber, J. W., Wanner, W. and Matthaeus, W. H.: Dominant twodimensional solar wind turbulence with implications for cosmic ray transport, J. Geophys. Res., 101, 2511-2522, 1996.

Bondeson, A.: Cascade properties of shear Alfvén turbulence, Phys. Fluids, 28, 2406-2411, 1985.

Bruno, R., Bavassano, B. and Villante, U.: Evidence for long period Alfvén waves in the inner solar system, J. Geophys. Res. 90, 4373-4377, 1985.

Bruno, R. and Bavassano, B.: Origin of low cross-helicity regions in the inner solar wind, J. Geophys. Res. 96, 7841-7851, 1991.

Bruno, R., Bavassano, B., Pietropaolo, E., Carbone, V., Veltri, P.: Effects of intermittency on interplanetary velocity and magnetic field fluctuations anisotropy, Geophys. Res. Lett. 26, 3185-3188, 1999.

Bruno, R., Carbone, V., Veltri, P., Pietropaolo, E. and Bavassano, B.: Identifying intermittency events in the solar wind, Planetary Space Sci., 49, 1201-1210, 2001.

Bruno, R., Carbone, V., Sorriso-Valvo, L., and Bavassano, B.: Radial evolution of solar wind intermittency in the inner heliosphere, J. Geophys. Res., 108 (A3), 1130, doi:10.1029/2002JA009615, 2003.

Bruno, R., Sorriso-Valvo, L., Carbone, V. and Bavassano, B.: Europhys. Lett., A possible truncated-Lvy-flight statistics recovered from interplanetary solar-wind velocity and magnetic-field fluctuations, 66, 146-152, 2004.

Burlaga, L.: Intermittent turbulence in the solar wind, J. Geophys. Res. 96, 5847-5851, 1991.

Carbone, V., Veltri, P., and Bruno, R.: Experimental evidence for differences in the extended self-silimarity scaling laws between fluid and magnetohydrodynamic turbulent flows, Phys. Rev. Lett. 75, 3110-3120, 1995.

Chang, T.: Self-organized criticality, multi-fractal spectra, and intermittent merging of coherent structures in the magnetotail, Astrophys. Space Sci. 264, 303-316, 1999.

Chang, T., Tam, S. W. Y., and Wu, C.: Phys. Plasmas, 11, 12871299, 2004.

Coleman, P. J.: Turbulence, Viscosity, and Dissipation in the SolarWind Plasma, Astrophys. J., 153, 371-383, 1968.

Farge, M., Holschneider, M., and Colonna, J. F.: Wavelet analysis of coherent structures in two-dimensional turbulent flows, in Topological Fluid Mechanics, ed. H.K. Moffat, Cambridge: Cambridge University Press, 765-776, 1990.

Goldstein, M. L., Roberts, D. A., and Matthaeus, W. H.: Magnetohydrodynamic Turbulence In The Solar Wind, Annual Rep. on Astron. and Astrophys., 33, 283-326, 1995.

Ho, C. M., Tsurutani, B. T., Goldstein, B. E., Phillips, J. L., and Balogh, A.: Tangential Discontinuities at high heliographic latitudes $\left(\sim-80^{\circ}\right)$, Geophys. Res. Lett., 22, 3409-3412, 1995.
Horbury, T. S., Balogh, A., Forsyth, R. J., \& Smith, E. J., ULYSSES observations of intermittent heliospheric turbulence, Advances in Space Research, 19, 847-850, 1997.

Klein, L., Bruno, R., Bavassano, B., and Rosenbauer, H.: Anisotropy and minimum variance of magnetohydrodynamic fluctuations in the inner heliosphere, J. Geophys. Res., 98, 17 461-17 466, 1993.

Kolmogorov, A. N.: The local structure of turbulence in incompressible viscous fluid for very large Reynolds numbers, C. R. Akad. Sci. SSSR 30, 301-341, 1941.

Kolmogorov, A. N.: A refinement of previous hypotheses concerning the local structure of turbulence in viscous incompressible fluid at high Reynolds number, J. Fluid mech. 177, 133-166., 1962.

Kraichnan, R. H.: Inertial-range spectrum of hydromagnetic turbulence, Phys. Fluids 8, 1385-1387, 1965.

Malara, F., Primavera, L., and Veltri, P.: Nonlinear evolution of parametric instability of a large-amplitude nonmonochromatic Alfvn wave, Phys. Plasmas, 7, 2866-2877, 2000.

Malara, F., Primavera, L., and Veltri, P.: Nonlinear evolution of the parametric instability: numerical predictions versus observations in the heliosphere, Nonlinear Proc. in Geophys., 8, 159166, 2001.

Mantegna, R. and Stanley, E. H.: Stochastic process with ultraslow convergence to a Gaussian: The truncated Lvy flight, Phys. Rev. Lett., 73, 2946-2949, 1994.

Marsch, E. and Liu, S.: Structure functions and intermittency of velocity fluctuations in the inner solar wind, Ann. Geophys. 11, 227-238, 1993.

Matthaeus, W. H., Goldstein, M. L., and Roberts, D. A.: Evidence for the presence of quasi-two-dimensional nearly incompressible fluctuations in the solar wind, J. Geophys. Res. 95, $20673-$ $20683,1990$.

Matthaeus, W. H. and Ghosh, S.: Spectral decomposition of solar wind turbulence: Three-component model, In S. Habbal, R. Esser, J. V. Hollweg, and P. A. Isenberg, editors, Solar Wind Nine, AIP Conf. Publ., 471, 519-526, 1999.

McCracken, K.G. and Ness, N. F.: The collimation of cosmic rays by the interplanetary magnetic field, J. Geophys. Res., 71, 3315$3325,1966$.

Nakagawa, T., Nishida, A., and Saito, T.: Planar magnetic structures in the solar wind, J. Geophys. Res. 94, 11 761-11 775, 1989.

Onorato, M., Camussi, R., and Iuso, G.: Anomalous scaling and bursting process in an experimental turbulent channel flow, Phys. Rev. E 61, 1447-1460, 2000.

Pagel C. and Balogh A.: Radial dependence of intermittency in the fast polar solar wind magnetic field using Ulysses, J. Geophys. Res., A108a.SSH2P, 2003.

Primavera, L., Malara, F., and Veltri, P.: Numerical Simulations of the Parametric Instability in Solar Wind, ESA SP-448, 11991204, 1999.

Primavera, L., Malara, F., and Veltri P.: Parametric instability in the solar wind: numerical study of the nonlinear evolution, paper presented at Solar Wind 10 Conference, 17-21 June 2002, Pisa, Italy, Eds. Velli, Bruno and Malara, AIP, 679, 505-508, 2003.

Riley, P., Sonett, C. P., Tsurutani, B. T., Balogh, A., Forsyth, R. J., and Hoogeveen, G. W.: Properties of arc-polarized Alfvn waves in the ecliptic plane: Ulysses observations, J. Geophys. Res., 101, 19987-19993, 1996.

Roberts, D. A., Goldstein, M. L., and Matthaeus W. H.: Velocity shear generation of solar wind turbulence, J. Geophys. Res. 97, $17115-17$ 130, 1992. 
Ruzmaikin, A., Feynman, J., Goldstein, B., and Balogh, A.: Intermittent turbulence in solar wind from the south polar hole, J. Geophys. Res. 100, 3395-3404, 1995.

Sagdeev, R. Z. and Galeev, A. A.: Nonlinear Plasma Theory, Ed. by O. Neil and D. Book, p. 7, W. A. Benjamin, New York, 1969.

Shebalin, J. V., Matthaeus, W. H., and Montgomery, D. C.: Anisotropy in MHD turbulence due to a mean magnetic field, D. J. Plasma Phys. 29, 525-547, 1983.

Sorriso-Valvo, L. , Carbone, V., Veltri, P., Consolini, G., and Bruno, R.: Intermittency in the solar wind turbulence through probability distribution functions of fluctuations, Geophys. Res. Lett. 26, 1801-1804, 1999.

Tsurutani, B. T., Ho, C. M., Smith, E. J., Neugebauer, M., Goldstein, B. E., Mok, J. S., Arballo, J. K., Balogh, A., Southwood, D. J., and Feldman, W. C.: The relationship between interplanetary discontinuities and Alfven waves: ULYSSES observations, Geophys. Res. Lett., 21, 2267-2270, 1994.

Tu, C.-Y and Marsch, E.: Evidence for a "background" spectrum of the solar wind turbulence in the inner heliosphere, J. Geophys. Res. 95, 4337-4341, 1990.

Tu, C.-Y, Marsch, E., and Thime, K. M.: Basic properties of solar wind MHD turbulence analysed by means of Elsësser variables, J. Geophys. Res. 95, 11739-11 759, 1989.

Tu, C.-Y and Marsch, E.: A case study of very low cross-helicity fluctuations in the solar wind, Ann. Geophys. 9, 319-332, 1991.
Tu, C.-Y and Marsch, E.: A model of solar wind fluctuations with two components: Alfvén waves and convective structures, J. Geophys. Res. 98, 1257-1276, 1993.

Tu, C.-Y and Marsch, E.: MHD structures, waves and turbulence in the solar wind: observations and theories, Space Sci. Rev., 73, 1-210, 1995.

Tu, C.-Y, Marsch, E., and Rosenbauer, H.: An extended structure function model and its aplication to the analysis of solar wind intermittency properties, Ann. Geophys. 14, 270-285, 1996.

Van Atta, C. W. and Park, J.: Statistical self-similarity and inertial subrange turbulence, Lect. Notes in Phys., 73, 402-426, 1975.

Vasquez, B. J. and Hollweg, J. V.: Nonlinear Alfvén waves. 1. Interactions between outgoing and ingoing waves according to an amplitude expansion, J. Geophys. Res., 109:A05103, doi:10.1029/2003JA010105, 2004.

Vasquez, B. J., Markovskii, S. A., and Hollweg, J. V.: Nonlinear Alfvén waves. 2. The influence of wave advection and finite wavelength effects., J. Geophys. Res., 109:A05104, doi:10.1029/2003JA010106, 2004.

Veltri, P. and Mangeney, A.: Scaling Laws and Intermittent Structures in Solar Wind MHD Turbulence, In S. Habbal, R. Esser, J. V. Hollweg, and P. A. Isenberg, editors, Solar Wind Nine, AIP Conf. Publ., 471, 543-546, 1999.

Wu, C. C and Chang, T.: 2D MHD Simulation of the Emergence and Merging of Coherent Structures, Geophys. Res. Lett., 27, 863-866, 2000. 